\title{
Universality Limits of a Reproducing Kernel for a Half-Line Schrödinger Operator and Clock Behavior of Eigenvalues
}

\author{
Thesis by \\ Anna Maltsev \\ In Partial Fulfillment of the Requirements \\ for the Degree of \\ Doctor of Philosophy
}

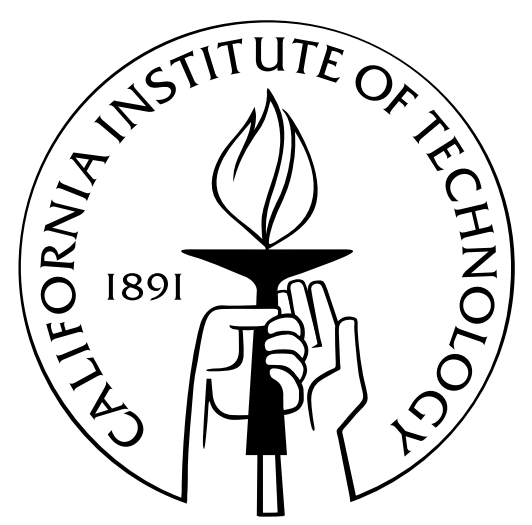

California Institute of Technology

Pasadena, California

2010

(Defended May 10, 2010) 
(C) 2010

Anna Maltsev

All Rights Reserved 
to my brother 


\section{Acknowledgements}

I would like to express my deepest gratitude to my advisor, Prof. Barry Simon, for his invaluable guidance and unflagging support.

I would also like to thank Jonathan Breuer and Fritz Gesztesy for useful discussions, Lilian Wong for proofreading drafts, the Caltech faculty for creating a wonderful environment to work in, and my friends and family for their love and support. 


\section{Abstract}

We extend some recent results of Lubinsky, Levin, Simon, and Totik from measures with compact support to spectral measures of Schrödinger operators on the half-line. In particular, we define a reproducing kernel $S_{L}$ for Schrödinger operators and we use it to study the fine spacing of eigenvalues in a box of the half-line Schrödinger operator with perturbed periodic potential. We show that if solutions $u(\xi, x)$ are bounded in $x$ by $e^{\epsilon x}$ uniformly for $\xi$ near the spectrum in an average sense and the spectral measure is positive and absolutely continuous in a bounded interval $I$ in the interior of the spectrum with $\xi_{0} \in I$, then uniformly in $I$

$$
\frac{S_{L}\left(\xi_{0}+a / L, \xi_{0}+b / L\right)}{S_{L}\left(\xi_{0}, \xi_{0}\right)} \rightarrow \frac{\sin \left(\pi \rho\left(\xi_{0}\right)(a-b)\right)}{\pi \rho\left(\xi_{0}\right)(a-b)},
$$

where $\rho(\xi) d \xi$ is the density of states. We deduce that the eigenvalues near $\xi_{0}$ in a large box of size $L$ are spaced asymptotically as $\frac{1}{L \rho}$. We adapt the methods used to show similar results for orthogonal polynomials. 


\section{Contents}

Acknowledgements iv

$\begin{array}{lll}\text { Abstract } & \text { v }\end{array}$

1 Introduction 1

1.1 Intro . . . . . . . . . . . . . . . . . . . . 1

1.2 Notations . . . . . . . . . . . . . . . . . . . . 1

1.3 Results ........................... 4

1.4 Methods ........................ 6

$\begin{array}{lll}2 & \text { Background } & 10\end{array}$

2.1 Introduction . . . . . . . . . . . . . . . . . . . . . 10

2.2 Schrödinger operators . . . . . . . . . . . . . . . . . 10

2.2.1 Periodic Schrödinger Operator: Floquet Solutions and the Discriminant ....................... . . 10

2.2 .2 Density of States . . . . . . . . . . . . . . 11

2.2.3 Asymptotics of Solutions and Gap Sizes for Periodic Schrödinger Operators .............................. 12

2.2.4 The Green's Function . . . . . . . . . . . . . . . . . . 13

2.2.5 Herglotz functions and the m-function . . . . . . . . . . 14

2.3 Orthogonal Polynomials . . . . . . . . . . . . . . . . . . . 16

2.3 .1 Introduction . . . . . . . . . . . . . . . . . 16

2.3.2 Regularity . . . . . . . . . . . . . . . 17

2.4 Reproducing Kernels . . . . . . . . . . . . . . . . . . . . . . . . . . . 18 
vii

2.4.1 The Reproducing Kernel for Orthogonal Polynomials . . . . . 18

2.4.2 Paley-Wiener Space and the Reproducing Kernel for Schrödinger Operators ............................... 18

2.4.3 Variational Principle . . . . . . . . . . . . . . . . . 20

2.4.4 Christoffel-Darboux Formula . . . . . . . . . . . . . 20

2.5 Universality and Clock Behavior _. . . . . . . . . . . . . 21

2.5.1 History of Universality Problems in Orthogonal Polynomials . 21

2.5.2 Lubinsky's Results and Methods . . . . . . . . . . . 22

2.5.3 Clock Behavior for Orthogonal Polynomials . . . . . . . . . 22

2.5.4 Spacing of Eigenvalues for Schrödinger Operators . . . . . . . 24

3 Proofs of Main Results 26

3.1 The Perturbed Periodic Potential . . . . . . . . . . . . . . 26

3.2 Variational Principle and the Christoffel-Darboux Formula . . . . . . 30

3.3 Bounds on the Diagonal Kernel . . . . . . . . . . . . . . . . . . . . . 35

3.4 Calculation of the reproducing kernel in the case of a periodic potential 41

3.5 Off-Diagonal Kernel and Clock Behavior . . . . . . . . . . . . . . 46

3.6 Example: the Free Schrödinger Operator . . . . . . . . . . . . . . . . 50

Bibliography $\quad 52$ 


\section{Chapter 1}

\section{Introduction}

\section{$1.1 \quad$ Intro}

In this thesis we exploit the similarities between differential and difference equations to show a half-line Schrödinger operator analogue of recent results of Lubinsky, Levin, Simon, and Totik. In this paper we provide definitions of a reproducing kernel $S_{L}$ and of regularity for half-line Schrödinger operators. We prove the analogues of universality and clock behavior of eigenvalues in a box for perturbed periodic halfline Schrödinger operators.

\subsection{Notations}

Let

$$
\tau \phi(x)=-\frac{d^{2} \phi(x)}{d x^{2}}+V(x) \phi(x)
$$

be a differential expression. We assume throughout that $V$ is locally integrable and bounded from below. Let $u, y$ be the standard fundamental solutions of

$$
\tau \phi(\xi, x)=\xi \phi(\xi, x)
$$

with initial conditions

$$
u(\xi, 0)=1=y^{\prime}(\xi, 0), u^{\prime}(\xi, 0)=0=y(\xi, 0) .
$$


Throughout the paper, $u^{\prime}, y^{\prime}$ denote the derivative with respect to $x$.

Associated to $\tau$ is a Schrödinger operator $A$ in $L^{2}([0, \infty))$ given by

$$
\begin{gathered}
A \phi=\tau \phi \\
\phi \in \operatorname{dom} A=\left\{\phi \in \mathrm{E}^{2}: \phi, \phi^{\prime} \in A C([0, c]) \forall c \in(0, \infty) ; \phi(0)=0\right\},
\end{gathered}
$$

where $A C([a, b])$ are the absolutely continuous functions on $[a, b]$, as in [GZ06]. This operator is then maximal, as shown in Chap. 3 of [Wei87]. Our results are valid for both Dirichlet and Neumann boundary conditions, but we only give the proofs for the Neumann case.

The adjoint operator $A^{*}$ of a densely defined linear operator $A$ is defined by

$$
\begin{gathered}
D\left(A^{*}\right)=\{\psi \in H: \exists \tilde{\psi} \in H,\langle\psi, A \phi\rangle=\langle\tilde{\psi}, \phi\rangle \forall \phi \in D(A)\} \\
A^{*} \psi=\tilde{\psi},
\end{gathered}
$$

and an operator $A$ is self-adjoint if $A=A^{*}$ and $D(A)=D\left(A^{*}\right)$. Since $V$ is assumed to be bounded from below, Theorem 1.1 of [BS91] gives that $A$ is essentially self-adjoint, and therefore there exists a unique self-adjoint extension (Section 2.2 [Tes09]).

There is a shift of notation here from the orthogonal polynomials literature, so $x$ in our setup is analogous to $n$ of the discrete case, and our $\xi$ is the analogue of $x$ of the discrete case. The analogues are illustrated in the following "translation" table: 
3

\begin{tabular}{|c|c|}
\hline Difference & Differential \\
\hline recurrence relation & differential expression \\
orthogonal polynomials & solutions of eigenvalue equation \\
$x^{n}$ & $\cos (\sqrt{\xi} x)$ \\
$x^{n}+a_{n-1} x^{n-1}+\ldots$ & $\int_{0}^{L} f(x) \cos (\sqrt{\xi} x) d x$ with $f \in L^{2}(0, \infty]$ \\
$x$ & $\xi$ in $u(\xi, x)$ \\
$n$ & $\mathrm{x}$ in $u(\xi, x)$ \\
\hline
\end{tabular}

The spectral measure of an operator $A$ is given by Theorem 2.2.3 of [Mar86] as follows. It is a measure $d \mu$ which makes the following two formulas hold for every function $f \in L^{2}[0, \infty)$ :

$$
\begin{aligned}
W(\zeta, f) & =\int_{0}^{\infty} f(x) u(\zeta, x) d x \\
f(x) & =\int W(\zeta, f) u(\zeta, x) d \mu(\zeta)
\end{aligned}
$$

and

$$
\langle f, g\rangle_{d m}=\langle W(\zeta, f), W(\zeta, g)\rangle_{d \mu(\zeta)} .
$$

Its existence is guaranteed by the theorem. We change variables from Marchenko so that his $\sqrt{\xi}$ is our $\xi$. In other words, for the Neumann boundary condition, there is a unitary transform $U: L^{2}([0, \infty)) \rightarrow L^{2}(d \mu)$, given by integration against the solution $u(\xi, x) d x$. The inverse transform is given by integration against $u(\xi, x) d \mu(\xi)$. These transforms are isometries. We let $\mathfrak{e}=\sigma_{\text {ess }}(A)$.

We now define a reproducing kernel $S_{L}$ for Schrödinger operators.

Definition 1.2.1. Given a Schrödinger operator $A$ as in (1.2.4) with the Neumann 
boundary condition, we let the reproducing kernel be

$$
S_{L}(\xi, \zeta)=\int_{0}^{L} u(\xi, t) u(\zeta, t) d t
$$

We see that the reproducing property is satisfied with respect to $d \mu$ :

$$
u(\xi, x) \chi_{[0, L]}(x)=\int S_{L}(\xi, \zeta) u(\zeta, x) d \mu(\zeta)
$$

We are primarily interested in the case where the potential $V=q+p$ where $p$ is periodic with period $P$ and continuous.

Definition 1.2.2. We call a perturbation q non-destructive if it leaves the essential spectrum unchanged and zero-average if

$$
\frac{1}{x} \int_{0}^{x}|q(t)| d t \rightarrow 0
$$

We assume throughout that the perturbation $q$ is a non-destructive zero-average perturbation e.g. $q \rightarrow 0$ at $\infty$.

The spectrum of a periodic Schrödinger operator $\mathfrak{e}$ is a union of closed intervals (Background Section 2.2.1). Let $\Delta(\xi)=y(\xi, P)+u^{\prime}(\xi, P)$ be the discriminant and let $\mathfrak{e}=\cup\left[l_{n}, r_{n}\right]$ so that $\Delta$ is a invertible on each $\left[l_{n}, r_{n}\right]$. We call each $\left[l_{n}, r_{n}\right]$ a band and each interval in $\mathbb{R} \backslash \mathfrak{e}$ a gap. When $r_{n}=l_{n+1}$, we call the point $\xi=r_{n}$ a closed gap. Furthermore, there exists a first band, so shifting $q$ by a constant in energy, we can assume that $\min \mathfrak{e}=0$.

We let $A=-\frac{d^{2}}{d x^{2}}+p(x)+q(x)$ be our perturbed periodic Schrödinger operator, where $p$ is continuous periodic potential and $q$ is the perturbation.

\subsection{Results}

We can now state our main result:

Theorem 1.3.1. Let $A=-\frac{d^{2}}{d x^{2}}+p(x)+q(x)$ with periodic and continuous $p$ and non-destructive zero-average $q$ and let $d \mu(\xi)=w(\xi) d \xi+d \mu_{s}$ be its spectral measure. 
Let $I \subset \mathfrak{e}^{\text {int }}$ be a closed and bounded interval such that $w$ is continuous and non-zero on $I$ and $\operatorname{supp}\left(d \mu_{s}\right) \cap I=\emptyset$. Let $\xi_{0} \in I$ and $a, b, B \in \mathbb{R}$. Then uniformly in $I$ and $|a|,|b|<B$

$$
\frac{S_{L}\left(\xi_{0}+a / L, \xi_{0}+b / L\right)}{S_{L}\left(\xi_{0}, \xi_{0}\right)} \rightarrow \frac{\sin \left(\pi \rho\left(\xi_{0}\right)(a-b)\right)}{\pi \rho\left(\xi_{0}\right)(a-b)},
$$

where $\rho(\xi) d \xi$ is the density of states.

Like in the discrete case, the asymptotic behavior of the kernel $S_{L}$ for the perturbed periodic operator $A$ depends on the density of states $\rho(\xi) d \xi$ of the periodic operator $A^{\#}$, defined, for example, in Berezin-Shubin (see Background Section 2.2.2). The measure $\rho(\xi) d \xi$ is the same for Dirichlet and Neumann boundary conditions.

It is well known that for the Christoffel-Darboux kernel

$$
K_{n}(x, y)=\frac{\gamma_{n-1}}{\gamma_{n}} \frac{p_{n}(x) p_{n-1}(y)-p_{n-1}(x) p_{n}(y)}{x-y}
$$

where $p_{n}$ 's are orthonormal polynomials and $\gamma_{n}$ is the leading coefficient as in [ST92]. This expression is called the Christoffel-Darboux formula (Background Section 2.4.4), and we show its analogue in Section 3.2 for $S_{L}$.

From (1.3.1) and the Christoffel-Darboux formula (3.2.4) we deduce that the zeros of $u^{\prime}(-, L)$, scaled by the density of states, will be asymptotically equally spaced, like the zeros of the sine function. We adapt the definition from [LS08] (Background Section 2.5.3):

Definition 1.3.2. Fix $\xi^{*}$ in an interval I, and number the zeros $\xi_{N}$ of $u^{\prime}(-, L)$ with increasing positive integers to the right of $\xi^{*}$ and decreasing negative integers to the left so that ... $<\xi_{-1}<\xi^{*} \leq \xi_{0}<\ldots$. We say there is strong clock behavior of zeros of $u^{\prime}$ at $\xi^{*}$ on an interval $I$ if the density of states $\rho(\xi) d \xi$ is continuous and nonvanishing on $I$ and for fixed $n$

$$
\lim _{L \rightarrow \infty} L\left|\left(\xi_{n}-\xi_{n+1}\right)\right| \rho\left(\xi^{*}\right)=1
$$

and we say there is uniform clock behavior on I if the limit in (2.5.3) is uniform on $I$ for fixed $n$. 
In Section 3.5, we show

Corollary 1.3.3. Let $A, \mathfrak{e}, I, \xi_{0}$ as in Theorem 1.3.1. Then there is uniform clock behavior of the zeros of $u^{\prime}$ and $y$ on $I$.

As an example we consider the case $p=0$. In Section 3.6 we show by direct computation that given same conditions on the measure as in Theorem 1.3.1 we have

$$
\lim _{L \rightarrow \infty} \frac{S_{L}\left(\xi+\frac{a}{L}, \xi+\frac{b}{L}\right)}{S_{L}(\xi, \xi)}=\frac{\sin \left(\frac{a-b}{2 \sqrt{\xi}}\right)(2 \sqrt{\xi})}{a-b}
$$

which yields that the eigenvalues in a box of size $L$ are spaced asymptotically as $\frac{1}{2 L \sqrt{\xi}}$.

\subsection{Methods}

Regularity is a key property of measures on compact support, and has received a lot of attention in OP literature (Background Section 2.3.2). We adapt the regularity condition to spectral sets of half-line Schrödinger operators as follows:

Definition 1.4.1. Suppose $\mathfrak{e} \subset \mathbb{R}$ is the essential support of a spectral measure $d \mu$

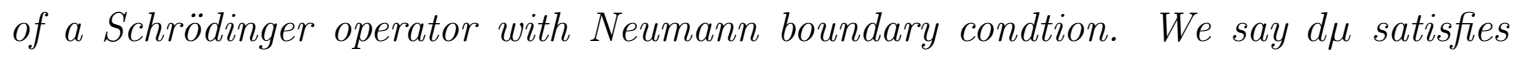
regularity bounds if for any $\epsilon>0$ there exists $\delta_{1}>0, C$ such that for all $\xi$ with $\operatorname{dist}(\xi, \mathfrak{e}) \leq \delta_{1}$ the solution $u$ satisfies

$$
\int_{0}^{L} u(\xi, x)^{2} d x \leq C e^{\epsilon L}
$$

with $C$ not dependent on $\xi, L$.

In Section 3.1 we show that a Schrödinger operator with potential of the form $q(x)+p(x)$ with continuous periodic $p$ and non-destructive zero-average $q$ (as in Definition 1.2.2) satisfies regularity bounds.

Lubinsky's inequality carries over to our setup, as we show in Section 3.5. Given two measures $d \mu \leq d \mu^{*}$, this inequality gives us a bound on the difference of offdiagonal kernel in terms of ratios of diagonal kernels. Then similar to Lubinsky 
[Lub09], we use the variational principle (see Background Section 2.4.3) to get asymptotics of ratios of diagonal kernels. We find a model measure, for which we can show universality directly. In our case it is the periodic Schrödinger operator. We construct a comparison measure that dominates both the model measure and the measure of interest. Then we use our version of Lubinsky's inequality (3.5.1) to compare the kernels of the measure of interest to the comparison measure and the kernels of the model measure to the comparison measure. Then we deduce that the asymptotics of the model measure kernel and that of the measure of interest are close to the same thing (namely, the asymptotics of the kernel of the comparison measure), they are close to each other. Since we know the universality for the model measure, we thus get it for the measure of interest as well.

Similar to Simon [Sim08b] and Lubinsky [Lub09], we need a measure $d \mu^{\#}(\xi)=$ $w^{\#}(\xi) d \xi+d \mu_{s}^{\#}$, which corresponds to a Schrödinger operator $A^{\#}$ and satisfies the following properties (we call such a measure a model)

(1) $\sigma_{\mathrm{ess}}\left(\mu^{\#}\right)=\mathfrak{e}$

(2) $w^{\#}$ is continuous and nonvanishing on $\mathfrak{e}$

(3) For any compact interval $I \subset \mathfrak{e}^{\text {int }}$ and $\epsilon>0$ as $L \rightarrow \infty$ uniformly on $I$

$$
\sup _{\xi \in I} e^{-\epsilon L} S_{L}\left(\xi, \xi, d \mu^{\#}\right) \rightarrow 0 .
$$

(4) For any compact interval $I \subset \mathfrak{e}^{\text {int }}$ for all $\xi \in I$ uniformly,

$$
\lim _{\epsilon \rightarrow 0} \lim _{L \rightarrow \infty} \frac{S_{L+\epsilon L}\left(\xi, \xi, \mu^{\#}\right)}{S_{L}\left(\xi, \xi, \mu^{\#}\right)}=1 .
$$

(5) For $\xi(L) \rightarrow \xi_{0}$ in $\mathfrak{e}^{\text {int }}$

$$
\lim _{L \rightarrow \infty} \frac{S_{L}(\xi(L), \xi(L))}{S_{L}\left(\xi_{0}, \xi_{0}\right)}=1
$$

and this limit is uniform in $I$. 
We need these properties in the proof of Theorem 1.4.2. Theorem 3.4.3 immediately implies that the operator $A^{\#}$ with periodic potential satisfies model conditions 3-4. In Theorem 3.4.1, we notice that model condition 5 is satisfied. Thus, $A^{\#}$ is a model. We therefore can use the periodic potential as a model for $\mathfrak{e}$, whenever $q$ is non-destructive.

We construct the comparison measure $d \mu^{*}$ to dominate both $d \mu$ and $d \mu^{\#}$ and to be continuous and non-vanishing on $I$ with $w^{*}\left(\xi_{0}\right)=w\left(\xi_{0}\right)$. We let $d \mu^{*}$ be the sup of $d \mu, d \mu^{\#}$ on a compact subset of $\mathbb{R}$ and $d \mu+d \mu^{*}$ on the rest of $\mathbb{R}$. The comparison measure is a scalar multiple of a spectral measure, as we show in Section 3.5. We call such measures unnormalized spectral measures, as analogous to unnormalized measures on compact sets. If $u, y$ is a fundamental system of solutions and $S_{L}$ the reproducing kernel associated to a spectral measure $d \mu$, then for $s>0$ we associate $\frac{u}{\sqrt{s}}, \frac{y}{\sqrt{s}}$, and the reproducing kernel $\frac{1}{s} S_{L}(\zeta, \xi, d \mu(\xi))$ to $d(s \mu)$. A spectral measure $d \mu$ must have a prescribed asymptotic at infinity (Theorem 2.4.2 of [Mar86]), which implies that the normalization constant $s$ is unique and the reproducing kernel is well-defined. Henceforward, we use the letters $d \mu, d \mu^{*}$ to denote spectral measures which may be unnormalized and all results in Section 3.2 are shown for unnormalized spectral measures. Also, the definition of regularity bounds works just as well.

The perturbed operator may have countably many eigenvalues in each gap, but the only limit points are the bands' endpoints. When $p$ is bounded and continuous, the size of the $n$th gap goes to 0 as $n \rightarrow \infty$ (Lemma 2.9 of [MW66]), so only finitely many gaps and finitely many eigenvalues do not lie in $\left\{\xi: \operatorname{dist}(\xi, \mathfrak{e}) \leq \delta_{1}\right\}$ for any $\delta_{1}>0$. By construction, the same will be true for the comparison measure. We end up needing this fact in the proof of Lemma 3.3.3.

In Section 3.3, we show

Theorem 1.4.2. Suppose $d \mu(\xi)=w(\xi) d \xi+d \mu_{s}, d \mu^{*}(\xi)=w^{*}(\xi) d \xi+d \mu_{s}^{*}$ are unnormalized spectral measures with $\sigma_{\mathrm{ess}}(d \mu)=\sigma_{\mathrm{ess}}\left(d \mu^{*}\right)=\mathfrak{e}$. Suppose $d \mu$, d $\mu^{*}$ satisfy regularity bounds and have finitely many eigenvalues outside of $\left\{\xi: \operatorname{dist}(\xi, \mathfrak{e})<\delta_{1}\right\}$ for any $\delta_{1}>0$. Suppose that at least one of $d \mu, d \mu^{*}$ is a model measure. Let $I \subset \mathfrak{e}^{\text {int }}$ be a closed and bounded interval such that $w, w^{*}$ are continuous and strictly positive 
on $I$ and $\left(\operatorname{supp}\left(d \mu_{s}\right) \cup \operatorname{supp}\left(d \mu_{s}^{*}\right)\right) \cap I=\emptyset$. Let $\xi_{0} \in I$ and $\xi(L) \rightarrow \xi_{0}$ as $L \rightarrow \infty$. Then uniformly in I

$$
\frac{S_{L}(\xi(L), \xi(L), \mu)}{S_{L}\left(\xi(L), \xi(L), \mu^{*}\right)} \rightarrow \frac{w^{*}\left(\xi_{0}\right)}{w\left(\xi_{0}\right)}
$$

In Section 3.4, we compute the universality limit of the kernel in the unperturbed periodic case to be

$$
\lim _{L \rightarrow \infty} \frac{S_{L}\left(\xi_{0}+\frac{a}{L}, \xi_{0}+\frac{b}{L}\right)}{S_{L}\left(\xi_{0}, \xi_{0}\right)}=\frac{\sin \left(\pi \rho\left(\xi_{0}\right)(a-b)\right)}{\pi \rho\left(\xi_{0}\right)(a-b)}
$$

where $\rho(\xi) d \xi$ is the density of states corresponding to the periodic Schrödinger operator. To make this calculation, we use a standard formula to express the density of states in terms of the imaginary part of the diagonal Green's function, and then we express the Green's function in terms of the solution $u$.

From Theorem 1.4.2 and adapted Lubinsky's inequality we deduce Theorem 1.3.1. 


\section{Chapter 2}

\section{Background}

\subsection{Introduction}

Before we get to the proofs of the main results, we give brief introductions into the several areas used. In Section 2.2, we give an overview of spectral theory of Schrödinger operators, focusing particularly on the periodic case and its perturbations. In Section 2.3, we give a brief introduction into the area of orthogonal polynomials, since we use a lot of their methods. In Section 2.4, we give some details of the theory of reproducing kernels, as it features prominently in our work. In Section 2.5, we give some history of the problem.

\section{$2.2 \quad$ Schrödinger operators}

\subsubsection{Periodic Schrödinger Operator: Floquet Solutions and the Discriminant}

Here we review the theory of the periodic Schrödinger operator. The analysis of periodic Schrödinger operators dates back to Bloch and Floquet. Most of the material presented here can be found in [RS78] and [BS91].

Consider a differential operator $\tau$ with periodic potential with period $P$. One notes that the translation operator $T$ given by $T \psi(x)=\psi(x+P)$ maps the solution space of the differential equation $\tau \phi=\xi \phi$ to itself. We want to diagonalize $T$, so we 
solve the equation $\psi(x+P)=e^{i \theta P} \psi(x)$ so that the solution $\psi$ can be written as a product $\psi(\xi, x)=e^{i \theta x} \phi(\xi, x)$, where $\phi(\xi, x)$ is periodic.

Since $u(\xi, x+P), y(\xi, x+P)$ satisfy the same differential equation and $u(\xi, x)$, $y(\xi, x)$ form a basis of the solution space, we can write

$$
\begin{gathered}
u(\xi, x+P)=c_{11} y(\xi, x)+c_{12} u(\xi, x) \\
u(\xi, x+P)=c_{21} y(\xi, x)+c_{22} u(\xi, x) .
\end{gathered}
$$

Substituting the boundary condition at 0 into the above and into the same system differentiated with respect to $x$ and solving for $c_{i j}$, we get

$$
c_{11}=y(\xi, P), c_{12}=y^{\prime}(\xi, P), c_{21}=u(\xi, P), c_{22}=u^{\prime}(\xi, P) .
$$

The matrix of $T$ in the $u, y$ basis is called the monodromy matrix, and by the constancy of the Wronskian, its determinant is 1 . The characteristic equation for the eigenvalues $s$ of $T$ becomes

$$
s^{2}-\Delta(\xi) s+1=0
$$

where $\Delta(\xi)=y(\xi, P)+u^{\prime}(\xi, P)$. Then we need $|s|=1$ so that the solutions of the differential equation are not exponentially growing [Sim82], which happens only when $|\Delta(\xi)|<2$, so $\overline{\{\xi:|\Delta(\xi)| \leq 2\}}$ is the spectrum. One can furthermore show that $\Delta^{\prime}(\xi) \neq 0$ whenever $\Delta(\xi) \in(-2,2)$.

\subsubsection{Density of States}

If we restrict our periodic operator $A^{\#}$ with period $P$ to $L^{2}[0, n P]$ and consider a periodic boundary condition (either Neumann or Dirichlet), it will have a countable discrete set of eigenvalues. For an interval $(a, b)$, let $N_{n P}((a, b))$ be the number of eigenvalues in the interval $(a, b)$. Then the limit

$$
\lim _{n \rightarrow \infty} \frac{N_{n P}((a, b))}{n P}=\int \rho(\xi) d \xi
$$


exists and defines an absolutely continuous measure on $\mathbb{R}$ called the density of states. Here $\rho$ turns out to be a non-negative locally integrable function [BS91].

\subsubsection{Asymptotics of Solutions and Gap Sizes for Periodic Schrödinger Operators}

Proposition 2.2.1. Let $A u=-\frac{d^{2}}{d x^{2}} u+V(x) u$ be a Schrödinger operator with $V$ bounded. Then

$$
\begin{aligned}
& |u(\xi, x)| \leq e^{\frac{M x}{\sqrt{\xi}}} \\
& |y(\xi, x)| \leq e^{\frac{M x}{\sqrt{\xi}}} .
\end{aligned}
$$

Proof. One can show this proposition using the method of successive approximations; see for example [MW66] and [CS77]. Let

$$
\begin{aligned}
& u_{n}(x, \xi)=\frac{1}{\sqrt{\xi}} \int_{0}^{x} V(t) u_{n-1}(t) \sin ((x-t) \sqrt{\xi}) d t \\
& y_{n}(x, \xi)=\frac{1}{\sqrt{\xi}} \int_{0}^{x} V(t) y_{n-1}(t) \sin ((x-t) \sqrt{\xi}) d t
\end{aligned}
$$

and

$$
\begin{aligned}
& u_{0}(x)=\cos (\sqrt{\xi} x) \\
& y_{0}(x)=\sin (\sqrt{\xi} x)
\end{aligned}
$$

Then $u=\sum u_{n}$ is the solution of $A$. One can check this by taking two derivatives, and substituting the sum into the original equation. It remains to check that the sums are absolutely convergent. We show by induction that $\left|u_{n}\right| \leq \frac{(M x)^{n}}{\sqrt{\xi^{n}} n !}$, where $M=\sup |V(x)|$. Clearly, $u_{0} \leq 1$. We assume that

$$
\left|u_{n-1}\right| \leq \frac{(M x)^{n-1}}{\sqrt{\xi}^{n-1}(n-1) !}
$$


Then

$$
\begin{aligned}
\left|u_{n}\right| & =\left|\frac{1}{\sqrt{\xi}} \int_{0}^{x} V(t) u_{n-1}(t) \sin ((x-t) \sqrt{\xi}) d t\right| \\
& \leq \frac{1}{\sqrt{\xi}} \int_{0}^{x}\left|V(t) \frac{(M t)^{n-1}}{\sqrt{\xi}^{n-1}(n-1) !} \sin ((x-t) \sqrt{\xi})\right| d t \\
& \leq \frac{M^{n}}{\sqrt{\xi}^{n}(n-1) !} \int_{0}^{x} t^{n-1}=\frac{(M x)^{n}}{\sqrt{\xi}^{n} n !}
\end{aligned}
$$

Similarly, $\left|y_{n}\right| \leq \frac{(M x)^{n}}{\sqrt{\xi}^{n} n !}$ for $\xi>1$.

Both sums converge absolutely for all $x$, and recalling the Taylor series for the exponential function the proposition follows.

Further details can be found in [MW66]. We need this proposition to show regularity bounds for perturbed periodic Schrödinger operator. The method of proof of this proposition can also be used to prove the following proposition:

\section{Proposition 2.2.2.}

$$
\lim _{\xi \rightarrow \infty} \sqrt{\xi}(\Delta(\sqrt{\xi})-2 \cos \pi \sqrt{\xi})=0
$$

We need the following fact about gap sizes, and this is weaker than Lemma 2.9 of [MW66].

Lemma 2.2.3. When $p$ is bounded, the size of the $n$th gap goes to 0 as $n \rightarrow \infty$.

This follows from (2.2.5).

\subsubsection{The Green's Function}

The Green's function associated to a differential operator is its fundamental solution with the given boundary condition. If $A$ is our Schrödinger operator with the Neumann boundary condition, then $(A-z) G\left(z, x, x^{\prime}\right)=\delta\left(x-x^{\prime}\right)$ in $x$, and $\frac{d}{d x} G\left(z, 0, x^{\prime}\right)=$ 0, i.e. $G$ satisfies the same boundary condition. Equivalently, the Green's function is the integral kernel of the resolvent of $A$, i.e. $\left((A-z)^{-1} f\right)\left(x^{\prime}\right)=\int_{0}^{\infty} G\left(z, x, x^{\prime}\right) f(x) d x$. 
We assume that our operator is limit point, meaning that exactly one solution for each complex $z$ is in $L^{2}$. We call this solution $\psi$, and we normalize it as $\psi^{\prime}(z, 0)=$ 1. Given the fundamental solutions $u$ and $y$ of $A$ and the solution $\psi$, the Green's function with the Neumann boundary condition at the origin will be given piecewise by $G\left(z, x, x^{\prime}\right)=u(z, x) \psi\left(z, x^{\prime}\right)$ for $0 \leq x \leq x^{\prime}$ and $G\left(z, x, x^{\prime}\right)=u\left(z, x^{\prime}\right) \psi(z, x)$, otherwise, as given in [CL55]. Similarly, for Dirichlet boundary condition, we use $y$ instead of $u$ in the same formula.

There is a standard formula to express the density of states in terms of the imaginary part of the diagonal Green's function [Rei04], which we use in Section 3.4. Let $A_{L}$ be the restriction of the operator $A$ to $L^{2}([0, L])$. Then if $\xi_{A_{L}, n}$ are the eigenvalues for $A_{L}$, the trace of the resolvent will be given by

$$
\operatorname{Tr}\left(z-A_{L}\right)^{-1}=\sum_{n} \frac{1}{z-\xi_{n}} .
$$

Furthermore,

$$
\lim _{\epsilon \downarrow 0} \frac{1}{\xi-\xi_{n}+i \epsilon}=\Re\left(\frac{1}{\xi-\xi_{n}}\right)-i \pi \delta\left(\xi-\xi_{n}\right),
$$

so that

$$
\Im \lim _{\epsilon \downarrow 0} \operatorname{Tr}\left(A_{L}+\xi+i \epsilon\right)^{-1}=\sum \delta\left(\xi-\xi_{A_{L}, n}\right) .
$$

Linking the trace of the resolvent to the diagonal Green's function and taking limits in $L$ to get the density of states and letting $B \subset \mathbb{R}$,

$$
\int_{B} \rho(\xi) d \xi=\int_{B} \lim _{L \rightarrow \infty} \frac{1}{L} \sum \delta\left(\xi-\xi_{A_{L}, n}\right) d \xi=\int_{B} \lim _{L \rightarrow \infty} \lim _{\epsilon \downarrow 0} \frac{1}{L} \int_{0}^{L} \Im G(\xi+i \epsilon, x, x) d x d \xi
$$

\subsubsection{Herglotz functions and the m-function}

Here we give a different description of the spectral measure via the $m$-function. Most of the material summarized here can be found in [GZ06], [Tes09]. Let $A=-\frac{d^{2}}{d x^{2}}+$ $V(x)$ be a self-adjoint Schrödinger operator, and let $u$ and $y$ be the solutions of the differential equation $A \phi=z \phi$ corresponding to Neumann and Dirichlet boundary 
conditions respectively. Since $u$ and $y$ will span the solution space for each $z$, then the $L^{2}$ solution $\psi$ as defined in the previous section is given by

$$
\psi(z, x)=u(z, x)+m(z) y(z, x) .
$$

The $m(z)$ in the above equation is the definition of the Titchmarsh-Weyl $m$ function. One can show that the $m$-function is a Herglotz function, and the Herglotz inversion theorem yields a measure on $\mathbb{R}$, which happens to be the spectral measure of $A$. Here I will sketch a proof that appears in [Tes09]. First one shows that

$$
\Im(m(z))=\Im(z) \int_{0}^{\infty}|\psi(z, x)|^{2} d x
$$

using the well-known formula (can be checked directly) relating the integral of two solutions $\phi_{1}, \phi_{2}$ at different points to the Wronskian:

$$
\left(z_{1}-z_{2}\right) \int_{0}^{x} \phi_{1}\left(z_{1}, t\right) \phi_{2}\left(z_{2}, t\right) d t=W\left(\phi_{1}\left(z_{1}, x\right) \phi_{2}\left(z_{2}, x\right)\right)-W\left(\phi_{1}\left(z_{1}, 0\right) \phi_{2}\left(z_{2}, 0\right)\right) .
$$

Then we can use $\psi(z, x)$ and $\psi(\bar{z}, x)=\overline{\psi(z, x)}$ as our two solutions and $z, \bar{z}$ as our two points to get the desired result.

Recall there exists $U$ a unitary map $U: L^{2}[0, \infty) \rightarrow L^{2}(\mathbb{R}, d \mu)$, given by integration against $u(\xi, x) d x$ and the inverse given by integration against $u(\xi, x) d \mu(\xi)$. Furthermore, if $R_{A}(z)$ is the resolvent of $\mathrm{A}$,

$$
R_{A}(z) f=U^{-1} \frac{1}{\xi-z} U f
$$

SO

$$
\int_{0}^{\infty} G(z, x, y) f(y) d y=\int \frac{u(\xi, x) F(\xi)}{\xi-z} d \mu(\xi)
$$

where $F=U f$. At $x=0$, substituting for the Green's function and by continuity of 
both sides in $x$, we obtain

$$
\int_{0}^{\infty} \psi(z, y) f(y) d y=\int \frac{F(\xi)}{\xi-z} d \mu(\xi)
$$

for all compactly supported $F$. Since such $F$ are dense. we get that

$$
U \psi(z)(\xi)=\frac{1}{\xi-z}
$$

Since $U$ is an isometry, we get that

$$
\Im(m(z))=\Im(z) \int_{0}^{\infty}|\psi(z, x)| d x=\Im(z) \int \frac{1}{|\xi-z|^{2}} d \mu(\xi) .
$$

Since

$$
\Im\left(\frac{1}{\xi-z}-\frac{\xi}{1+\xi}\right)=\frac{\Im(z)}{|\xi-z|^{2}}
$$

$m(z)$ is the Herglotz transform of the spectral measure $d \mu$, so the Herglotz inversion theorem gives us the $d \mu$.

\subsection{Orthogonal Polynomials}

\subsubsection{Introduction}

Let $d \eta=w(x) d x+d \eta_{s}$ be a probability measure supported on a compact set $\mathfrak{e}$. Polynomials form a Hilbert space with $L^{2}(d \eta)$ inner product, and the set $\left\{1, x, x^{2}, \ldots\right\}$ forms a basis. We can obtain a set of orthonormal polynomials $p_{n}$ by the GramSchmidt algorithm. One can furthermore show that orthonormal polynomials $p_{n}$ satisfy a three-term recurrence relation, i.e. for suitable $\left\{a_{n}, b_{n}\right\}_{n=1}^{\infty}$,

$$
x p_{n}(x)=a_{n+1} p_{n+1}(x)+b_{n+1} p_{n}(x)+a_{n} p_{n-1}(x) .
$$

This three term recurrence relation gives us a tridiagonal matrix, called the Jacobi matrix, which, given a boundary condition, gives an operator on $l^{2}$. For a given $x$, 
the vector $p_{n}(x)$ is the solution of the corresponding the eigenvalue equation with the boundary condition $p_{0}(x)=1$.

The recurrence relation also gives the leading coefficient $\gamma_{n}$ of the orthonormal polynomials in terms of $a_{n}$ 's: $\gamma_{n}=\left(a_{n} a_{n-1} \ldots a_{1}\right)^{-1}$.

\subsubsection{Regularity}

A definition of regularity is most natural in terms of logarithmic capacity of the support of the measure, call it $C(\mathfrak{e})$. One can show that for any measure with compact support $\mathfrak{e}$

$$
\lim \sup \gamma_{n}^{-1 / n} \leq C(\mathfrak{e})
$$

so it is natural to study the class of measures for which equality holds. Such measures are called regular, and they satisfy several equivalent properties. Firstly, it is equivalent to root asymptotics of orthogonal polynomials i.e.

$$
\lim _{n \rightarrow \infty}\left|p_{n}(d \mu, z)\right|=e^{g(z, \infty)}
$$

for all $z$ outside of the convex hull of the support of $d \eta$. Regularity implies the existence of an equilibrium measure, and the converse is almost true as well, up to some degenerate cases. Additionally, regularity is usually an assumption on the measure in proofs of universality of the reproducing kernel, as in [Lub09]. It is for this last capacity that we seek to define an analogue of regularity for Schrödinger operators.

Regular measures are described in detail in [ST92]. For the interval, Erdos-Turan [ET55] show the relationship between root asymptotics, the existence of equilibrium measures, and positivity of the weight. Ullman [Ull72] then studied systematically regularity and asymptotics of orthogonal polynomials for arbitrary measures supported on $[-1,1]$.

Since spectral measures are supported on unbounded sets, no useful notion of capacity is possible. An equivalent capacity-free definition of regularity that we adapt 
to our purposes is the following. The measure $d \eta$ on a compact set $\mathfrak{e}$ is regular [ST92] if for any $\epsilon>0$ there exist $\delta>0$ and a constant $C$ so that

$$
\sup _{\operatorname{dist}(y, \mathfrak{e}) \leq \delta}\left|p_{n}(y, d \eta)\right| \leq C e^{\epsilon n}
$$

\subsection{Reproducing Kernels}

\subsubsection{The Reproducing Kernel for Orthogonal Polynomials}

The Christoffel-Darboux kernel $K_{n}$, given by

$$
K_{n}(x, y)=\sum_{k=0}^{n} p_{k}(x) p_{k}(y)
$$

(see for example [ST92], [Lub09], [Sim08a]), is characterized by the reproducing property, i.e. for all $k<n$,

$$
p_{k}(y)=\int K_{n}(x, y) p_{k}(x) d \eta(x)
$$

This is easy to see: interchanging the sum and the integral, each term except $k$ th is 0 , by orthonormality.

\subsubsection{Paley-Wiener Space and the Reproducing Kernel for Schrödinger Operators}

In our setup, we use the space

$$
H_{L}=\left\{\pi: \pi(\xi)=\int_{0}^{L} f(x) \cos (\sqrt{\xi} x) d x, f \in L^{2}[0, \infty)\right\}
$$

as the analogue of the space of polynomials with degree less than or equal to $n$.

The orthogonal polynomials with degree smaller than or equal to $n$ are a basis for the space of polynomials with degree less than or equal to $n$. The analogous property of $H_{L}$ is

$$
H_{L}=\left\{\pi: \pi(\xi)=\int_{0}^{L} f(x) u(\xi, x) d x, f \in L^{2}[0, \infty)\right\}
$$


This follows easily from Marchenko (see (1.2.10), (1.2.10") of [Mar86]), which gives the existence of a continuous integral kernel $M$, such that

$$
\pi(\xi)=\int_{0}^{L} f(x)\left(u(\xi, x)+\int_{0}^{x} M(x, t) u(\xi, t) d t\right) d x .
$$

The parameter $L$ is analogous to the polynomial degree. Here is how:

Proposition 2.4.1. Let $\pi \in H_{L}$ and $\sigma \in H_{N}$. If $\pi * \sigma \in L^{2}$, then $\pi \sigma \in H_{L+N}$.

Proof. Let $\pi(\xi)=\int_{0}^{L} f(x) \cos (\sqrt{\xi} x) d x$ and $\sigma(\xi)=\int_{0}^{N} g(y) \cos (\sqrt{\xi} x) d y$, then

$$
\pi(\xi) \sigma(\xi)=\int_{0}^{L} \int_{0}^{N} f(x) g(y) \cos (\sqrt{\xi} x) \cos (\sqrt{\xi} y) d x d y
$$

Let $\tilde{f}, \tilde{g}$ be even functions with $f=\tilde{f} \chi_{[0, L]}$ and $g=\tilde{g} \chi_{[0, N]}$, then the Fourier sine transform of $\tilde{f}$ and $\tilde{g}$ is 0 and

$$
\begin{aligned}
\pi(\xi) \sigma(\xi) & =1 / 2 \int_{-L}^{L} \int_{-N}^{N} \tilde{f}(x) \tilde{g}(y) e^{i \sqrt{\xi}(x+y)} d x d y \\
& =1 / 2 \int_{-L-N}^{L+N} \int_{-N}^{N} \chi_{[-L, L]}(u-x) \tilde{f}(x) \tilde{g}(u-x) d x e^{i \sqrt{\xi} u} d u
\end{aligned}
$$

Since $\sigma * \pi \in L^{2}$, so is $\tilde{f} * \tilde{g}$. Suffices to show that $\int_{-N}^{N} \chi_{[-L, L]}(u-x) \tilde{f}(x) \tilde{g}(u-x) d x$ is symmetric in $u$ :

$$
\begin{gathered}
\int_{-N}^{N} \chi_{[-L, L]}(u-x) \tilde{f}(x) \tilde{g}(u-x) d x=\int_{-N}^{N} \chi_{[-L, L]}(-u+x) \tilde{f}(-x) \tilde{g}(-u+x) d x= \\
=\int_{-N}^{N} \chi_{[-L, L]}(-u-x) \tilde{f}(x) \tilde{g}(-u-x) d x
\end{gathered}
$$

by the change of variables $x \mapsto-x$.

The space of polynomials of degree less than or equal to $n$ is usually considered with the $L_{2}(d \eta)$ inner product. Analogously, we give $H_{L}$ the following inner product:

$$
\left\langle\pi_{1}, \pi_{2}\right\rangle=\int \pi_{1}(\zeta) \overline{\pi_{2}(\zeta)} d \mu(\zeta)
$$


where $d \mu$ is the spectral measure.

\subsubsection{Variational Principle}

The minimizer of $\|\pi(y)\|_{L^{2}(d \eta)}$ over polynomials $\pi$ with $\operatorname{deg} \pi \leq n$ and $\pi(x)=1$ is

equal to $\frac{K_{n}(x, y)}{K_{n}(x, x)}$ and the minimum is equal to $K_{n}(x, x)^{-1}$. This property is called the variational principle and we show its analogue for $S_{L}$ :

Theorem 2.4.2. If $\mu$ is an unnormalized spectral measure, then

$$
\min \left\{\|Q\|_{d \mu}: Q \in H_{L}, Q\left(\xi_{0}\right)=1\right\}=S_{L}\left(\xi_{0}, \xi_{0}\right)^{-1}
$$

and the minimizer is given by

$$
\frac{S_{L}\left(\xi, \xi_{0}\right)}{S_{L}\left(\xi_{0}, \xi_{0}\right)}
$$

We give the minimum its own letter:

$$
\lambda_{L}(\xi)=S_{L}(\xi, \xi)^{-1}
$$

\subsubsection{Christoffel-Darboux Formula}

This formula is very well-known and useful. In particular it is used to show interlacing of zeros of orthogonal polynomials, and the link between universality and clock behavior of zeros, as discussed later. One can find a discussion of the ChristoffelDarboux formula in Simon's 1.9 of [Sim05]. If $K_{n}(x, y)$ is the reproducing kernel as before and $\left\{a_{n}, b_{n}\right\}$ are the coefficients of the corresponding two-term recurrence, then

$$
K_{n}(x, y)=a_{n+1} \frac{p_{n+1}(x) p_{n}(y)-p_{n}(x) p_{n+1}(y)}{x-y}
$$

To see this, one lets

$$
Q_{j}(x, y)=a_{j}\left(p_{j}(x) p_{j-1}(y)-p_{j}(y) p_{j-1}(x)\right)
$$


Then note that

$$
(x-y) p_{j}(x) p_{j}(y)=Q_{j+1}(x, y)-Q_{j}(x, y)
$$

Taking the sum over $j$ and doing appropriate cancelations, we get the desired result.

A similar formula holds for reproducing kernels of Schrödinger operators, as we show in Section 3.2.

\subsection{Universality and Clock Behavior}

\subsubsection{History of Universality Problems in Orthogonal Poly- nomials}

Kuijlaars-Vanlessen [KV02] use Riemann-Hilbert techniques to obtain universality limits for generalized Jacobi weights, both in the bulk and at the edge. Lubinsky [Lub09] shows universality for weights on $(-1,1)$ under much weaker hypotheses than used previously. In particular, there is no requirement of analyticity on the weight as in [KV02]. This result is interesting for both the study of orthogonal polynomials and of random matrices. It relates a fundamental object to the sine kernel and implies that the left hand side of (2.5.1) only depends on the continuity and positivity of the measure $d \eta$ at $x_{0}$ and its essential support. Simon [Sim08b] and Totik [Tot] extend this argument to measures with $\operatorname{supp}_{\text {ess }}(d \eta)=\cup I_{j}$ a finite union of intervals. In this thesis I adapt all the steps to Schrödinger operators.

Universality with variable weights was studied a lot in the context of random matrices. It in dates back to Dyson in the 60's, making the hypothesis of universality for all unitary, orthogonal, and symplectic ensembles. In random matrix theory, a reproducing kernel arises as a two point correlation function of the point process that describes the eigenvalue distribution. A survey of the random matrix literature can be found in [KV02]. 


\subsubsection{Lubinsky's Results and Methods}

Let $I \subset(-1,1)$ be a closed interval and $d \eta$ is regular such that $\operatorname{supp}\left(d \mu_{s}\right) \cap I=\emptyset$ and $w$ is continuous and nonvanishing on $I$. Then Lubinsky [Lub09] shows that for $a, b \in \mathbb{R}$ and uniformly for $x_{0} \in I$

$$
\lim _{n \rightarrow \infty} \frac{K_{n}\left(x_{0}+\frac{a}{n}, x_{0}+\frac{b}{n}\right)}{K_{n}\left(x_{0}, x_{0}\right)}=\frac{\sin \left(\pi \rho_{[-1,1]}\left(x_{0}\right)(a-b)\right)}{\pi \rho_{[-1,1]}\left(x_{0}\right)(a-b)}
$$

where $\rho_{[-1,1]}\left(x_{0}\right)=\left(\pi \sqrt{1-x_{0}^{2}}\right)^{-1}$ is the density of states for $[-1,1]$.

We summarize Lubinsky's method for showing (2.5.1). He notes that if $d \eta, d \eta^{*}$ are regular measures on $[-1,1]$ with $d \eta \leq d \eta^{*}$ and $K^{*}$ is the Christoffel-Darboux kernel associated with $d \eta^{*}$,

$$
\frac{\left|K_{n}(x, y)-K_{n}^{*}(x, y)\right|}{K_{n}(x, x)} \leq\left(\frac{K_{n}(y, y)}{K_{n}(x, x)}\right)^{1 / 2}\left(1-\frac{K_{n}^{*}(x, x)}{K_{n}(x, x)}\right)^{1 / 2}
$$

This inequality, called Lubinsky's inequality, implies that in order to understand the left hand side of (2.5.1), it is sufficient to understand $K_{n}^{\#}(x, y)$ for some model measure $d \eta^{\#}$ and the behavior of a ratio of diagonal kernels. A model $d \eta^{\#}$ with $w^{\#}\left(x_{0}\right)=w\left(x_{0}\right)$ is chosen, for which $K_{n}^{\#}(x, y)$ can be computed directly. Then $d \eta^{*}=$ $\sup \left\{d \eta^{\#}, d \eta\right\}$ dominates both $d \eta$ and $d \eta^{\#}$ and a has similarly nice local behavior at $x_{0}$ with $w^{*}\left(x_{0}\right)=w\left(x_{0}\right)$. By the variational principle, the ratios of the diagonal kernels $\frac{K_{n}^{\#}(x, x)}{K_{n}^{*}(x, x)}$ and $\frac{K_{n}(x, x)}{K_{n}^{*}(x, x)}$ both converge to 1 , and Lubinsky's inequality and a comparison of the two resulting expressions yields the desired result.

\subsubsection{Clock Behavior for Orthogonal Polynomials}

The equilibrium measure $d \eta=\rho(x) d x$ gives us an estimate of the number of zeros of $p_{n}$ in a given interval for any large $n$. This gives that the estimate on the distance between two consecutive zeros near $x^{*}$ is $\rho\left(x^{*}\right)^{-1}$. If we multiply all zeros of $p_{n}$ by $\rho$, the average distance between them will be 1 . One then seeks the distribution of the distances between consecutive zeros. 
If we view zeros of $p_{n}$ as a point process on the line, one can distinguish two extreme cases. The zeros might not "interact" at all, i.e. be distributed as a Poisson process, or they can "repel each other," i.e. be equally spaced. Both cases are possible under different conditions. Minami [Min96] shows Poisson behavior for some ergodic Jacobi matrices. Stoiciu in his dissertation shows Poisson behavior under similar conditions for the case of orthogonal polynomials on the unit circle. Recently, AvilaJitomirskaya show that something in between can also occur, in particular, for the the almost Mathieu operator with $\lambda>1$ and Diophantine coupling.

A precise definition of clock behavior for the zeros of orthogonal polynomials is given in [LS08].

Definition 2.5.1. Fix $x^{*}$ in an interval $I$, and number the zeros $x$ of $p_{n}$ with increasing positive integers to the right of $x$ and decreasing negative integers to the left so that $\ldots<x_{-1}<x^{*} \leq x_{0}<\ldots$. We say there is strong clock behavior of zeros at $x^{*}$ on an interval $I$ if the density of states $\rho(x) d x$ is continuous and nonvanishing on $I$ and for fixed $k$

$$
\lim _{n \rightarrow \infty} n\left|\left(x_{k}-x_{k+1}\right)\right| \rho\left(x^{*}\right)=1
$$

and we say there is uniform clock behavior on I if the limit in (2.5.3) is uniform on $I$ for fixed $k$.

This nomenclature comes from the theory of orthogonal polynomials on the unit circle. There, when zeros of polynomials exhibit clock behavior, they do indeed look like marks on a clock.

Szego in [Sze75] shows clock behavior for Jacobi polynomials, and Erdos-Turan [ET55] for a more general class of measures on [-1,1]. Last-Simon in [LS08] show clock behavior of zeros of orthogonal polynomials on the real line under weak conditions namely when Jacobi parameters approach the free ones and are of bounded variation.

In [Fre71], Freud notes that universality implies clock behavior. Levin-Lubinsky in [LL08] have a similar result. They use the interlacing property and the ChristoffelDarboux formula, and we adapt their proof to our setup. Here is a sketch of their 
proof: We number the zeros near a point $x^{*}$ as before. By the Christoffel-Darboux formula (3.2.4),

$$
\frac{p_{n}\left(x^{*}\right)}{p_{n-1}\left(x^{*}\right)}=\frac{p_{n}\left(x^{*}+a / n\right)}{p_{n-1}\left(x^{*}+a / n\right)}
$$

for $a \neq 0$ if and only if $K_{n}\left(x^{*}, x^{*}+a / n\right)=0$. Given universality, and since $K_{n}\left(x^{*}, x^{*}\right)=O(n), K_{n}\left(x^{*}, x^{*}+a / n\right)=o(1 / n)$ if and only if $a=\frac{k}{\rho\left(x^{*}\right)}+o(1 / n)$. Our proof of clock spacing for eigenvalues of the halfline Schrödinger equation follows a similar method.

\subsubsection{Spacing of Eigenvalues for Schrödinger Operators}

Similar to the discrete case, we scale the eigenvalues in a box for a Schrödinger operator and consider the distances between consecutive eigenvalues. We can once again distinguish the two cases of "no interactions" and "repulsion". In the former case, the scaled eigenvalues are a Poisson process, when viewed as a point process. In the latter case, the eigenvalues are asymptotically equally spaced. We say then there is clock behavior, and we give a precise definition of what that means in this thesis.

Poisson statistics for eigenvalues arise for some random potentials, and were first demonstrated by Molcanov [Mol81] in one dimension in the case of Brownian motion potentials. Later the proofs were much simplified and extended to multiple dimensions by Minami [Min96]. Molcanov considers restrictions of the original spectral problem to small intervals, thus "decoupling" the point process. There is a crucial difference between his setup and ours that leads to such different local behaviors of eigenvalues. In Molcanov's setup, there is no a.c. spectrum, while in ours we assume the existence of an interval $I$ where the spectrum is purely a.c.

If the potential of the Schrödinger operator is in $L^{1}$, Clock behavior of eigenvalues of Schrödinger operators follows almost immediately from Jost asymptotics of solutions. Jost introduced the notion of Jost solutions in the 40's in [Jos47], i.e. solutions which are asymptotically free. In particular, he shows that for $L^{1}$ potential, there 
exist two solutions $\psi_{+}$and $\psi_{-}$such that

$$
e^{\mp i \xi x} \psi_{ \pm}(\xi, x) \rightarrow 1
$$

as $x \rightarrow \infty$. Taking real and imaginary parts of $\psi_{+}$, we see that there are two solutions: one asymptotically sine, the other asymptotically cosine. The zeros of either will be asymptotically locally equally spaced, like the zeros of sine and cosine. Universality, as described in Section 2.5.1, 2.5.2 can be interpreted as miniature Jost asymptotics.

The spacing of eigenvalues for functions on $[0, L]$ with periodic Dirichlet boundary condition is the same as the spacing of zeros of $y(\xi, L)$ in $\xi$ in case of the Dirichlet boundary condition at 0 and $L$, since whenever $y\left(\xi_{0}, L\right)=0$ the periodic boundary condition is satisfied. Similar logic applies to the zeros of $u^{\prime}(\xi, L)$ in case of the Neumann boundary condition.

Levin-Lubinsky's proof that universality implies clock behavior of zeros for orthogonal polynomials requires the zeros of $p_{n}$ to interlace with the zeros of $p_{n-1}$. Similarly, in our proof, we need the roots of the solution to interlace with the roots of its derivative (in $x$ ). But if a solution $\psi$ and its derivative are both zero at some point $x_{0}$, then $\psi$ is the solution of the second order differential equation with the trivial boundary condition, and therefore must be identically 0 . 


\section{Chapter 3}

\section{Proofs of Main Results}

\subsection{The Perturbed Periodic Potential}

Let $\mathfrak{e}$ be the essential spectrum of a Schrödinger operator with period $P$ periodic potential $p$ and either Neumann or Dirichlet boundary condition. The goal of this section is to show

Proposition 3.1.1. A Schrödinger operator with essential spectrum $\mathfrak{e}$ and potential $V(x)=p(x)+q(x)$, where $p$ is periodic and continuous and $\frac{1}{x} \int_{0}^{x}|q(t)| d t \rightarrow 0$, satisfies regularity bounds.

Fix $\epsilon>0$ and let $\frac{1}{x} \int_{0}^{x}|p(t)+q(t)| d t \leq M$ for $x>x_{0}$, for some $x_{0}$. To prove (1.4.1), it is sufficient to show that $\int_{0}^{L} u(\xi, x)^{2} d x \leq C e^{\epsilon L}$ separately for three cases of $\xi$, where $C$ is uniform in $\xi, L$ :

(1) $\xi>\frac{4 M^{2}}{\epsilon^{2}}$, shown in Lemma 3.1.2

(2) $\xi \leq \frac{4 M^{2}}{\epsilon^{2}}, \xi$ in the interior of $\mathfrak{e}$, but slightly away from the endpoints of the intervals, i.e. $\xi \in\left(\cup\left[l_{n}+\epsilon, r_{n}-\epsilon\right]\right) \cap\left[0, \frac{4 M^{2}}{\epsilon^{2}}\right]$, shown in Lemma 3.1.3

(3) $\xi \leq \frac{4 M^{2}}{\epsilon^{2}}$ and $\xi$ near the interval endpoints i.e. $\xi \in\left(\cup\left[l_{n}-\epsilon, l_{n}+\epsilon\right] \cup\left[r_{n}-\epsilon, r_{n}+\right.\right.$ $\epsilon]) \cap\left[0, \frac{4 M^{2}}{\epsilon^{2}}\right]$, shown in Lemma 3.1.4

The three cases are illustrated in the following picture: 
Figure 3.1:

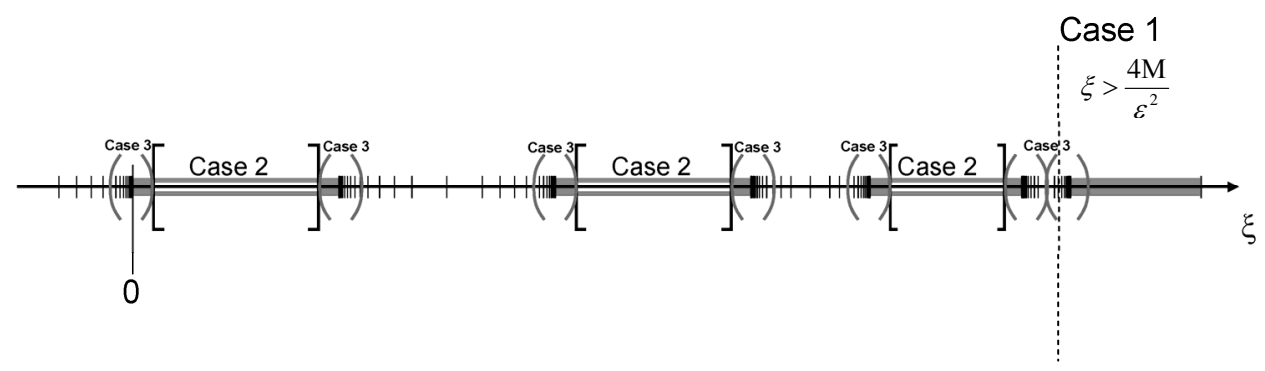

Lemma 3.1.2. Let $A=-\frac{d^{2}}{d x^{2}}+V(x)$ be a Schrödinger operator such that $\frac{1}{x} \int_{0}^{x}|V(t)| d t$ is bounded in $x$ as $x \rightarrow \infty$. Then the solutions $u, y$ of the eigenvalue equation satisfy

$$
\begin{aligned}
& |u(\xi, x)| \leq C e^{\frac{\int_{0}^{x}|V(t)| d t}{\sqrt{\xi}}} \\
& |y(\xi, x)| \leq C e^{\frac{\int_{0}^{x}|V(t)| d t}{\sqrt{\xi}}} .
\end{aligned}
$$

Proof. Using successive approximations, we can perturb about the solutions with $V=0$. Chadan-Sabatier ((I.2.3), (I.2.4), (I.2.6), (I.2.8a) [CS77]) show (3.1.2), and using $\cos (\sqrt{\xi} x)$ as initial data, instead of (I.2.3), gives (3.1.1).

This lemma indeed implies that for $\sqrt{\xi} \geq \frac{2 M}{\epsilon}$ the solution $u$ satisfies $u(x) \leq C e^{\frac{1}{2} \epsilon x}$, which implies $\int_{0}^{L} u(\xi, x)^{2} d x \leq C e^{\epsilon L}$.

Lemma 3.1.3. Let $\left[l_{n}, r_{n}\right]$ be a band of the spectrum for a Schrödinger operator $A=$ $-\frac{d^{2}}{d x^{2}}+q(x)+p(x)$ with periodic and continuous $p$ and non-destructive zero-average $q$ (Definition 1.2.2). Then the solution $u$ of the eigenvalue equation with the Neumann boundary condition satisfies $\int_{0}^{L} u(\xi, x)^{2} d x \leq C e^{\epsilon L}$ for $\xi \in\left(\cup\left[l_{n}+\epsilon, r_{n}-\epsilon\right]\right) \cap[0, R]$, where $R=\frac{4 M^{2}}{\epsilon^{2}}$, and same holds for the solution with the Dirichlet boundary condition.

Proof. Let $u_{p}(\xi, x), y_{p}(\xi, x)$ be the solutions of $A^{\#}=-\frac{d^{2}}{d x^{2}}+p(x)$ with boundary conditions

$$
\begin{aligned}
& u_{p}(\xi, 0)=1=y_{p}^{\prime}(\xi, 0) \\
& y_{p}(\xi, 0)=0=u_{p}^{\prime}(\xi, 0) .
\end{aligned}
$$


By Floquet's theorem (for example Section 1.2 of [MW66] and Theorem XIII.89 of [RS78]), there exists a solution $f(\xi, x)=e^{i \theta(\xi) x} \phi(\xi, x)$, where $\phi$ is periodic in $x$ with period $P$. We normalize $f^{\prime}(\xi, 0)=1$. The exponent $\theta(\xi)$ is not 0 or $\pi$ away from band endpoints, so that $\bar{f}$ is linearly independent of $f$ for $\xi \in \cup\left[l_{n}+\epsilon, r_{n}-\epsilon\right]$. Then

$$
u(\xi, x)=a_{1}(\xi) f(\xi, x)+a_{2}(\xi) \overline{f(\xi, x)} .
$$

We solve for $a_{1}, a_{2}$ in terms of $\xi$. We get that

$$
\begin{aligned}
& 1=u(\xi, 0)=a_{1}(\xi) f(\xi, 0)+a_{2}(\xi) \overline{f(\xi, 0)} \\
& 0=u^{\prime}(\xi, 0)=a_{1}(\xi) f^{\prime}(\xi, 0)+a_{2}(\xi) \overline{f^{\prime}(\xi, 0)}=a_{1}(\xi)+a_{2}(\xi),
\end{aligned}
$$

so that

$$
a_{1}(\xi)=-a_{2}(\xi)
$$

Substituting

$$
1=a_{1}(\xi) f(\xi, 0)-a_{1}(\xi) \overline{f(\xi, 0)}
$$

we get

$$
a_{1}(\xi)=(2 i \Im f(\xi, 0))^{-1}=-a_{2}(\xi)
$$

Since $f, \bar{f}$ are independent, $\Im f \neq 0$ and, by Theorem XIII.89 of [RS78], $f$ is analytic in $\xi$ on $\left[l_{n}+\epsilon, r_{n}-\epsilon\right]$. This implies that $a_{1}, a_{2}$ are analytic as well. The function $|f|$ is continuous in both $x$ and $\xi$ on $[0, P] \times\left(\cup\left[l_{n}+\epsilon, r_{n}-\epsilon\right] \cap[0, R]\right)$, therefore it achieves its maximum on this set. Since $|f|$ is periodic and continuous in $x$ with period $P$, the maximum of $|f|$ in $x$ for fixed $\xi$ occurs on $[0, P]$. This implies that $u_{p}(\xi, x) \leq K$, where $K$ is constant in $x$ and $\xi \in \cup\left[l_{n}+\epsilon, r_{n}-\epsilon\right] \cap[0, R]$.

We use the method of variation of parameters about $u_{p}(\xi,-), y_{p}(\xi,-)$ and Gronwall inequality. Let $d=u_{p}^{\prime} y_{p}-y_{p}^{\prime} u_{p} \neq 0$ be the Wronskian. We let

$$
\left(\begin{array}{c}
u(x) \\
u^{\prime}(x)
\end{array}\right)=\left(\begin{array}{ll}
u_{p}(x) & y_{p}(x) \\
u_{p}^{\prime}(x) & y_{p}^{\prime}(x)
\end{array}\right)\left(\begin{array}{l}
a(x) \\
b(x)
\end{array}\right)
$$


Then $a, b$ satisfy the differential equation

$$
\begin{aligned}
\left(\begin{array}{l}
a^{\prime}(x) \\
b^{\prime}(x)
\end{array}\right) & =d^{-1}\left(\begin{array}{cc}
-y_{p}\left(-u_{p}^{\prime \prime}+(q+p-\xi) u_{p}\right) & -y_{p}\left(-y_{p}^{\prime \prime}+(q+p-\xi) y_{p}\right) \\
u_{p}\left(-u_{p}^{\prime \prime}+(q+p-\xi) u_{p}\right) & u_{p}\left(-y_{p}^{\prime \prime}+(q+p-\xi) y_{p}\right)
\end{array}\right)\left(\begin{array}{c}
a(x) \\
b(x)
\end{array}\right) \\
& =\frac{q(x)}{d}\left(\begin{array}{cc}
-y_{p} u_{p} & -y_{p}^{2} \\
u_{p}^{2} & u_{p} y_{p}
\end{array}\right)\left(\begin{array}{c}
a(x) \\
b(x)
\end{array}\right)
\end{aligned}
$$

with the boundary condition $(a(0), b(0))=(1,0)$. This is equivalent to the integral equation

$$
\left(\begin{array}{l}
a(x) \\
b(x)
\end{array}\right)=\left(\begin{array}{l}
1 \\
0
\end{array}\right)+\int_{0}^{x} \frac{q(x)}{d}\left(\begin{array}{cc}
-y_{p} u_{p} & -y_{p}^{2} \\
u_{p}^{2} & u_{p} y_{p}
\end{array}\right)\left(\begin{array}{l}
a(t) \\
b(t)
\end{array}\right) d t
$$

If we let the norm of a matrix $M$ equal

$$
|M|=\sum\left|M_{i j}\right|
$$

we take the norm of both sides of the integral equation to get

$$
\begin{aligned}
\left|\left(\begin{array}{l}
a(x) \\
b(x)
\end{array}\right)\right| & =\left|\left(\begin{array}{l}
1 \\
0
\end{array}\right)+\int_{0}^{x} \frac{q(x)}{d}\left(\begin{array}{cc}
-y_{p} u_{p} & -y_{p}^{2} \\
u_{p}^{2} & u_{p} y_{p}
\end{array}\right)\left(\begin{array}{l}
a(t) \\
b(t)
\end{array}\right)\right| d t \\
& \leq 1+K_{1} \int_{0}^{x} q(x)\left|\left(\begin{array}{l}
a(t) \\
b(t)
\end{array}\right)\right| d t,
\end{aligned}
$$

where $K_{1} \geq\left|y_{p} u_{p}\right|+y_{p}^{2}+u_{p}^{2}+\left|u_{p} y_{p}\right|$ is constant in $x$ and $\xi$ by the argument above. We apply the Gronwall inequality to this integral equation to get

$$
|a(x)|+|b(x)| \leq K_{2} e^{K_{1} \int_{0}^{x}|q(t)| d x} .
$$

Then we take the matrix norm in (3.1) and, recalling that $\frac{1}{x} \int_{0}^{x}|q(t)| d t \rightarrow 0$, we get (1.4.1) for large $L$ and for all $L$ by choosing $C$ appropriately. 
Lemma 3.1.4. Let $\left[l_{n}, r_{n}\right]$ be a band of the spectrum for a Schrödinger operator $A=-\frac{d^{2}}{d x^{2}}+q(x)+p(x)$ with continuous periodic $p$ and non-destructive zero-average $q$ (Definition 1.2.2). Then the solution $u$ of the eigenvalue equation with Neumann boundary condition satisfies $\int_{0}^{L} u(\xi, x)^{2} d x \leq C e^{\epsilon L}$ for

$$
\xi \in\left(\cup\left[l_{n}-\epsilon, l_{n}+\epsilon\right] \cup\left[r_{n}-\epsilon, r_{n}+\epsilon\right]\right) \cap\left[0, \frac{4 M^{2}}{\epsilon^{2}}\right]
$$

The same holds for the solution with the Dirichlet boundary condition.

Proof. Let $\xi \in\left[l_{n}-\epsilon, l_{n}+\epsilon\right]$. We once again use the method of variation of parameters but this time about the solutions $u_{p}\left(-, l_{n}+\epsilon\right)$ and $y_{p}\left(-, l_{n}+\epsilon\right)$, i. e. the periodic solutions as before but at $\xi=l_{n}+\epsilon$ fixed. Like in the previous lemma, $u_{p}\left(x, l_{n}+\epsilon\right)$, $y_{p}\left(x, l_{n}+\epsilon\right)<K$, where $K$ is constant in $x$ and $\xi \in\left\{l_{n}, r_{n}\right\}_{n \in \mathbb{N}} \cap\left[0, \frac{4 M^{2}}{\epsilon^{2}}\right]$. We get

$$
\begin{aligned}
\left|\left(\begin{array}{l}
a(x) \\
b(x)
\end{array}\right)\right| & =\left|\left(\begin{array}{l}
1 \\
0
\end{array}\right)+\int_{0}^{x} \frac{l_{n}+\epsilon-\xi+q(x)}{d}\left(\begin{array}{cc}
-y_{p} u_{p} & -y_{p}^{2} \\
u_{p}^{2} & u_{p} y_{p}
\end{array}\right)\left(\begin{array}{l}
a(t) \\
b(t)
\end{array}\right) d t\right| \\
& \leq 1+K_{1} \int_{0}^{x}(2 \epsilon+|q(x)|)\left|\left(\begin{array}{c}
a(t) \\
b(t)
\end{array}\right)\right| d t
\end{aligned}
$$

As in proof of the previous lemma, applying Gronwall inequality and picking $C$ appropriately we get (1.4.1).

The three lemmas imply Proposition 3.1.1. From Lemma 3.1.2 we get (1.4.1) for large $\xi$. This leaves only finitely many bands, so it suffices to consider the remaining bands one at a time as in Lemmas 3.1.4 and 3.1.3.

\subsection{Variational Principle and the Christoffel-Darboux Formula}

We let $T_{L} F(\xi)=\int F(\zeta) S_{L}(\xi, \zeta) d \mu(\zeta)$, where $d \mu=d(s \nu)$ is a scalar multiple of a spectral measure $d \nu$. We show that $T_{L}$ is the orthogonal projection onto $H_{L}$. We first 
show

Lemma 3.2.1. The function $\cos (\sqrt{\xi} N)$ is fixed by $T_{L}$ for $N \leq L$.

Proof. Let $u$ be the solution associated to $d \mu$. There exists a continuous integration kernel $M$ ([GL55], (1.2.5") [Mar86]) such that

$$
\frac{\cos (\sqrt{\xi} x)}{\sqrt{s}}=u(\xi, x)+\int_{0}^{x} M(x, t) u(\xi, t) d t .
$$

Substituting this expression for $\frac{\cos (\sqrt{\xi} x)}{\sqrt{s}}$ in evaluating $T_{L}\left(\frac{\cos (\sqrt{\xi} x)}{\sqrt{s}}\right)$, we check

$$
\begin{gathered}
\int \frac{\cos (\sqrt{\xi} N)}{\sqrt{s}} S_{L}(\zeta, \xi) d \mu(\xi)=u(\xi, N)+\int_{0}^{N} M(N, t) \int u(\xi, t) S_{L}(\zeta, \xi) d \mu(\xi) d t= \\
=u(\xi, N)+\int_{0}^{N} M(N, t) u(\xi, t) d t=\frac{\cos (\sqrt{\xi} N)}{\sqrt{s}} .
\end{gathered}
$$

Here we use Fubini's theorem, the reproducing property of $S_{L}$ (noting that $N \leq L$ ), and we recover the last equality again by (3.2.1).

We then show that $T_{L}$ fixes $\pi_{N} \in H_{N}$ for $N \leq L$.

Corollary 3.2.2. If $\pi_{N}(\xi)=\int_{0}^{N} f(x) \cos (\sqrt{\xi} x) d x$ for some function $f \in L_{2}([0, N])$ and $N \leq L$, then $\pi_{N}(\xi)=\int \pi_{N}(\zeta) S_{L}(\xi, \zeta) d \mu(\zeta)$.

Proof. This is a straightforward calculation, using (3.2.1):

$$
\begin{gathered}
\int \pi_{N}(\zeta) S_{L}(\xi, \zeta) d \mu(\zeta)=\iint_{0}^{N} f(x) \cos (\sqrt{\zeta} x) S_{L}(\xi, \zeta) d x d \mu(\zeta)= \\
=\int_{0}^{N} f(x) \int \cos (\sqrt{\zeta} x) S_{L}(\xi, \zeta) d \mu(\zeta) d x=\pi_{N}(\xi)
\end{gathered}
$$

Here we make use of Fubini's theorem and the Lemma 3.2.1.

Theorem 3.2.3. The operator $\left(T_{L} \pi_{N}\right)(\xi)=\int \pi_{N}(\zeta) S_{L}(\xi, \zeta) d \mu(\zeta)$ is an orthogonal projection onto the Hilbert space $H_{L}$. 
Proof. To show that $T_{L}$ is a projection, by Corollary 3.2.2, it suffices to show that $T_{L} \pi_{N}(\xi) \in H_{L}$ for $N \geq L$. Recalling that $\pi_{N}(\xi)=\int_{0}^{N} f(x) \cos (\sqrt{\xi} x) d x$, we compute:

$$
\begin{gathered}
\int \pi_{N}(\zeta) S_{L}(\xi, \zeta) d \mu(\zeta)=\int d \mu(\zeta)\left(\int_{0}^{L}+\int_{L}^{N}\right) f(x) \cos (\sqrt{\zeta} x) S_{L}(\xi, \zeta) d x \\
=\pi_{L}(\xi)+\int d \mu(\zeta) \int_{L}^{N} f(x) \cos (\sqrt{\zeta} x) S_{L}(\xi, \zeta) d x
\end{gathered}
$$

We substitute $(3.2 .1)$ for $\cos (\sqrt{\zeta} x)$ to get

$$
\begin{aligned}
\int d \mu(\zeta) & \int_{L}^{N} f(x) \cos (\sqrt{\zeta} x) S_{L}(\xi, \zeta) d x= \\
& =\int d \mu(\zeta) \int_{L}^{N} f(x)\left(u(\zeta, x)+\int_{0}^{x} M(x, t) u(\zeta, t) d t\right) S_{L}(\xi, \zeta) d x
\end{aligned}
$$

We then use Marchenko's 1.2.5" to substitute for $\cos (\sqrt{\zeta} x)$ to get

$$
\begin{gathered}
\int d \mu(\zeta) \int_{L}^{N} f(x) \cos (\sqrt{\zeta} x) S_{L}(\zeta, \xi) d x= \\
=\int d \mu(\zeta) \int_{L}^{N} f(x)\left(u(\zeta, x)+\int_{0}^{x} M(x, t) u(\zeta, t) d t\right) S_{L}(\zeta, \xi) d x
\end{gathered}
$$

By Fubini and the reproducing property of the kernel, the first term is 0 . The second term is:

$$
\begin{gathered}
\int_{L}^{N} f(x) \int_{0}^{x} M(x, t) \int u(\zeta, t) S_{L}(\zeta, \xi) d \mu(\zeta) d t d x= \\
=\int_{L}^{N} f(x) \int_{0}^{x} M(x, t) u(\xi, t) \chi_{[0, L]}(t) d t d x= \\
=\int_{L}^{N} f(x) \int_{0}^{L} M(x, t) u(\xi, t)(t) d t d x \\
=\int_{0}^{L} \int_{L}^{N} f(x) M(x, t) d x u(\xi, t) d t .
\end{gathered}
$$

Letting $g(t)=\int_{L}^{N} f(x) M(x, t) d x$ we need only check that $\int_{0}^{L} g(t) u(\xi, t) d t \in H_{L}$, 
but this is clear from Marchenko's 1.2.10 which gives that

$$
u(\xi, t)=\cos (\sqrt{\xi} t)+\int_{0}^{t} K(t, y) \cos (\sqrt{\xi} y) d y
$$

Substituting, we get

$$
\begin{gathered}
\int_{0}^{L} g(t)\left(\cos (\sqrt{\xi} t)+\int_{0}^{t} K(t, y) \cos (\sqrt{\xi} y) d y\right) d t= \\
=\int_{0}^{L} g(t) \cos (\sqrt{\xi} t) d t+\int_{0}^{L} \int_{0}^{t} g(t) K(t, y) \cos (\sqrt{\xi} y) d y d t \\
=\int_{0}^{L} g(t) \cos (\sqrt{\xi} t) d t+\int_{0}^{L} \int_{y}^{L} g(t) K(t, y) d t \cos (\sqrt{\xi} y) d y \in H_{L}
\end{gathered}
$$

We next check that $T$ is self-adjoint:

$$
\begin{gathered}
\langle g, T f\rangle_{d(s \mu)}=\int d \mu(\xi) g(\xi) \int d \mu(\zeta) f(\zeta) S_{L}(\xi, \zeta)= \\
=\int d \mu(\zeta) f(\zeta) d \mu(\xi) g(\xi) S_{L}(\zeta, \xi)
\end{gathered}
$$

since our definition of $S_{L}$ is symmetric in $\zeta$ and $\xi$.

We now prove Theorem 2.4.2.

Proof. Fixing $\xi_{0} \in \mathbb{C}$ we consider

$$
\inf \left\{\|\pi\|^{2}: \pi_{L}(\xi)=\int_{0}^{L} f(x) \cos (\sqrt{\xi} x) d x ; \pi\left(\xi_{0}\right)=1\right\}
$$

If $\phi \neq 0$ is in some Hilbert space $H$, then

$$
\min \left\{\|\psi\|^{2}:\langle\psi, \phi\rangle=1\right\}=\frac{1}{\|\phi\|^{2}}
$$

and the minimizer is given by $\frac{\phi}{\|\phi\|^{2}}$ (Proposition 1.2.1 of [Sim05]). In our case, the 
Hilbert space is $H_{L}$. The condition that $\pi\left(\xi_{0}\right)=1$ is equivalent to

$$
1=\pi\left(\xi_{0}\right)=\int d \mu(\zeta) \pi(\zeta) S_{L}\left(\zeta, \xi_{0}\right)=\left\langle\pi, S_{L}\left(-, \xi_{0}\right)\right\rangle
$$

The proposition is applicable with $\phi(\xi)=S_{L}\left(\xi, \xi_{0}\right) \in H_{L}$ as shown above. Therefore the minimum is equal to

$$
\frac{1}{\left\|S_{L}\left(-, \xi_{0}\right)\right\|^{2}}
$$

We compute the $H_{L}$ norm in the first variable.

$$
\begin{aligned}
\left\|S_{L}\left(-, \xi_{0}\right)\right\|^{2} & =\iint_{0}^{L} u(\zeta, t) u\left(\xi_{0}, t\right) \int_{0}^{L} u(\zeta, x) u\left(\xi_{0}, x\right) d x d t d \mu(\zeta)= \\
& =\int_{0}^{L} u\left(\xi_{0}, t\right) \int u(\zeta, t) \int_{0}^{L} u(\zeta, x) u\left(\xi_{0}, x\right) d x d \mu(\zeta) d t= \\
& =\int_{0}^{L} u\left(\xi_{0}, t\right)^{2} d t=S_{L}\left(\xi_{0}, \xi_{0}\right),
\end{aligned}
$$

as desired. Furthermore, Simon 1.2.1 gives us the minimizer as

$$
S_{L}\left(\lambda, \lambda_{0}\right) / S_{L}\left(\lambda_{0}, \lambda_{0}\right)
$$

We show the analogue of the Christoffel-Darboux formula here:

\section{Lemma 3.2.4.}

$$
S_{L}(\alpha, \beta)=\frac{u(\alpha, L) u^{\prime}(\beta, L)-u(\beta, L) u^{\prime}(\alpha, L)}{\alpha-\beta}
$$

Proof.

$$
\begin{aligned}
& u(\alpha, x) u^{\prime \prime}(\beta, x)=u(\alpha, x)(q(x)-\beta) u(\beta, x) \\
& u(\beta, x) u^{\prime \prime}(\alpha, x)=u(\beta, x)(q(x)-\alpha) u(\alpha, x)
\end{aligned}
$$


We subtract to get

$$
u(\alpha, x) u^{\prime \prime}(\beta, x)-u(\beta, x) u^{\prime \prime}(\alpha, x)=(\alpha-\beta) u(\alpha, x) u(\beta, x) .
$$

Integrating both sides $d x$ from 0 to $L$, we get the desired formula. The left hand side has to be integrated by parts:

$$
\begin{aligned}
\int_{0}^{L} u(\alpha, x) u^{\prime \prime}(\beta, x) & -u(\beta, x) u^{\prime \prime}(\alpha, x) d x \\
& =u(\alpha, 0) u^{\prime}(\beta, 0)-u(\alpha, L) u^{\prime}(\beta, L)-u(\beta, 0) u^{\prime}(\alpha, 0)+u(\beta, L) u^{\prime}(\alpha, L) \\
& =u(\beta, L) u^{\prime}(\alpha, L)-u(\alpha, L) u^{\prime}(\beta, L)
\end{aligned}
$$

for any boundary condition given at 0 and independent of $\alpha, \beta$, such as Dirichlet or Neumann.

On the diagonal, the Christoffel-Darboux formula becomes

$$
S_{L}(\xi, \xi)=u^{\prime}(\xi, x) \frac{d}{d \xi} u(\xi, x)-\frac{d}{d \xi} u^{\prime}(\xi, x) u(\xi, x)
$$

\subsection{Bounds on the Diagonal Kernel}

We will show the analogue of Lemma 3.1 in Simon [Sim08b]. Assume regularity bounds (1.4.1) on the measure $d \mu$. Let

$$
Q_{L}\left(\xi, \xi_{0}\right)=\frac{S_{L}\left(\xi, \xi_{0}\right)}{S_{L}\left(\xi_{0}, \xi_{0}\right)}
$$

be the minimizer in $(3.2 .2)$.

Lemma 3.3.1. Let $d \mu$ be a measure that satisfies regularity bounds. Then for all $\epsilon>0$ there exist $C, \delta_{1}$ such that $\left|Q_{L}(\xi)\right| \leq C e^{\epsilon L} \lambda_{L}\left(\xi_{0}\right)$, for $\xi \in\left\{\xi: \operatorname{dist}(\xi, \mathfrak{e}) \leq \delta_{1}\right\}$

Proof. Fix $\epsilon . \quad$ A regularity bound (1.4.1) on a measure $d \mu$ implies a bound on 
$\left|S_{L}\left(\xi, \xi_{0}\right)\right|$ by Cauchy-Schwarz:

$$
\begin{aligned}
S_{L}\left(\xi, \xi_{0}\right) & =\left\langle u(\xi,-), u\left(\xi_{0},-\right)\right\rangle_{d m[0, L]} \\
& \leq\|u(\xi,-)\|\left\|u\left(\xi_{0},-\right)\right\| \\
& =\left(\int_{0}^{L} u(\xi, x)^{2} d x\right)^{1 / 2}\left(\int_{0}^{L} u\left(\xi_{0}, x\right)^{2}\right)^{1 / 2} \leq C e^{\epsilon L} .
\end{aligned}
$$

Dividing both sides by $S_{L}\left(\xi_{0}, \xi_{0}\right)$ gives the desired inequality.

To show Lemma 3.3.3 we need the following fact about the spectral measure:

Lemma 3.3.2. Let $A$ be a self adjoint Schrödinger operator and $d \mu$ be a scalar multiple of its spectral measure. Then for $n \geq 2$ there exists a constant $K$

$$
\int_{2}^{\infty} \frac{d \mu(\xi)}{\xi^{n}} \leq K 2^{-n}
$$

Proof. We give a proof for the Neumann boundary condition. For the Dirichlet boundary condition, a similar proof can be given using Section 6 of Gesztesy-Simon [GS00] for the asymptotic growth of the spectral measure.

By the product rule of differentiation,

$$
\frac{d \mu(\xi)}{\xi^{n}}=d\left(\frac{\mu(\xi)}{\xi^{n}}\right)+\frac{n \mu(\xi)}{\xi^{n-1}} d \xi
$$

We know from Marchenko's [Mar86] Theorem 2.4.2 that

$$
\lim _{\xi \rightarrow \infty} \mu(\xi)-\frac{2}{\pi} \xi=C
$$

This implies that for all $\epsilon_{1}$ there exists $R$ such that for all $\xi>R$,

$$
\left|\mu(\xi)-C-\frac{2}{\pi} \xi\right| \leq \epsilon .
$$


We take the integral from $R$ to $\tilde{R}$ in equation 3.3.3.

$$
\begin{aligned}
\int_{R}^{\tilde{R}} \frac{d \mu(\xi)}{\xi^{n}} & =\int_{R}^{\tilde{R}} d\left(\frac{\mu(\xi)}{\xi^{n}}\right)+\int_{R}^{\tilde{R}} \frac{n \mu(\xi)}{\xi^{n-1}} d \xi \\
& \leq \frac{\mu(\tilde{R})}{\tilde{R}^{n}}-\frac{\mu(R)}{R^{n}}+\int_{R}^{\tilde{R}} \frac{n(\xi+C+\epsilon)}{\xi^{n-1}} d \xi \\
& \leq \frac{\frac{2}{\pi} \sqrt{\tilde{R}}+C+\epsilon}{\tilde{R}^{n}}-\frac{\frac{2}{\pi} \sqrt{R}+C-\epsilon}{R^{n}}+\frac{n}{-n+3 / 2} \tilde{R}^{-n+3 / 2}-\frac{n}{-n+3 / 2} R^{-n+3 / 2}+ \\
& +(C+\epsilon) \frac{n}{-n+2} \tilde{R}^{-n+2}-(C+\epsilon) \frac{n}{-n+2} R^{-n+2}
\end{aligned}
$$

Taking $\tilde{R} \rightarrow \infty$ we get that

$$
\int_{R}^{\infty} \frac{d \mu(\xi)}{\xi^{n}} \leq K R^{-n+2}
$$

Now, the measure of the set $[2, R]$ is equal to $\mu(R)-\mu(2)<\infty$. So we get the desired bound on the integral

$$
\int_{2}^{R} \xi^{-n} d \mu \xi \leq 2^{-n} \mu([2, R])
$$

Lemma 3.3.3. Suppose $d \mu(\xi)=w(\xi) d \xi+d \mu_{s}, d \mu^{*}(\xi)=w^{*}(\xi) d \xi+d \mu_{s}^{*}$ are two unnormalized spectral measures with $\sigma_{\mathrm{ess}}(d \mu)=\sigma_{\mathrm{ess}}\left(d \mu^{*}\right)=\mathfrak{e}$. Suppose $d \mu$, d $\mu^{*}$ satisfy regularity bounds and have finitely many eigenvalues outside of $\left\{\xi: \operatorname{dist}(\xi, \mathfrak{e})<\delta_{1}\right\}$ for any $\delta_{1}>0$. Let $I \subset \mathfrak{e}^{\text {int }}$ be a closed and bounded interval such that $w, w^{*}$ are continuous and strictly positive on $I$ and $\left(\operatorname{supp}\left(d \mu_{s}\right) \cup \operatorname{supp}\left(d \mu_{s}^{*}\right)\right) \cap I=\emptyset$. Let $\xi_{0} \in I$ and $\xi(L) \rightarrow \xi_{0}$ as $L \rightarrow \infty$. Then for all sufficiently small $\delta$ and all $\epsilon>0$ and all $M$ there exist $\gamma<1, C$, $n$ such that for all $N>n+1$

$$
\lambda_{L}\left(\xi_{0}, \mu^{*}\right) \leq \sup _{\left|\xi-\xi_{0}\right|<\delta}\left(\frac{w^{*}(\xi)}{w(\xi)}\right) \lambda_{M}\left(\xi_{0}, \mu\right)+C e^{2 \epsilon M} \gamma^{N}+C e^{2 \epsilon M} 2^{-2 N}
$$


where $L=M+\frac{\pi}{4 \xi_{0}} N$.

Proof. We use the methods of Lubinsky [Lub09] and Simon [Sim08b].

Let $Q_{M}$ be the minimizing function for the measure $\mu$ and

$$
F(\xi)=\frac{4 \xi_{0}}{T \pi}\left(\frac{\sin \left(\frac{\pi}{4 \xi_{0}}\left(\xi-\xi_{0}\right)\right)}{\xi-\xi_{0}}+\frac{\sin \left(\frac{\pi}{4 \xi_{0}}\left(\xi+\xi_{0}\right)\right)}{\xi+\xi_{0}}\right)
$$

where $T=1+\frac{2}{\pi}$.

We notice that

(1) $\left|F\left(\xi_{0}\right)\right|=1$,

(2) $|F(\xi)|<\gamma$ whenever $\left|\xi-\xi_{0}\right| \geq \delta$, for some $0<\gamma<1$ depending on $\delta$, and

(3) $|F(\xi)|<\frac{C \xi_{0}}{\left|\xi-\xi_{0}\right|}$ whenever $\left|\xi-\xi_{0}\right|>1$.

The function $F$ is just $\frac{\sin (\xi)}{\xi}$ shifted so that 0 is at $\xi_{0}$, scaled so that exactly one period of the sine happens between 0 and $\xi_{0}$, then symmetrized to make it even, and then scaled by a factor of $\frac{1}{T}$ again to make $F\left(\xi_{0}\right)=1$. Since $\frac{\sin \xi}{\xi}=\int_{0}^{1} \cos (\xi x), F$ is a Fourier transform of some even function $f$ supported on $\left[-\frac{\pi}{4 \xi_{0}}, \frac{\pi}{4 \xi_{0}}\right]$, and $F^{N}$ is the Fourier transform of an even function with support in $\left[-\frac{N \pi}{4 \xi_{0}}, \frac{N \pi}{4 \xi_{0}}\right]$.

Fix $\epsilon$. Since the measures $d \mu$ and $d \mu^{*}$ are essentially supported on the same set $\mathfrak{e}$, we can let $\delta_{1}$ be as in the definition of regularity bounds (1.4.1) for both measures. Let $\mathfrak{e}_{\delta_{1}}=\left\{\xi: \operatorname{dist}(\xi, \mathfrak{e})<\delta_{1}\right\}$. We label the mass points of $d \mu^{*}$ outside $\mathfrak{e}_{\delta_{1}}$ with $\left\{\xi_{1}, \xi_{2}, \xi_{3}, \ldots, \xi_{n}\right\}$. We can construct a polynomial $P$ with zeros at $\xi_{1}, \ldots, \xi_{n}$ and a local maximum at $\xi_{0}$ of $P\left(\xi_{0}\right)=1$ with degree $n+1$.

Then let

$$
Q(\xi)=Q_{M}\left(\xi, \xi_{0}, \mu\right) F^{N} P
$$

Since $Q\left(\xi_{0}\right)=1$, by the minimizing property of $\lambda_{L}$,

$$
\|Q\|_{H_{L}\left(d \mu^{*}\right)}^{2} \geq \lambda_{L}\left(\xi_{0}, \mu^{*}\right)
$$


We then find a bound on $\|Q\|_{H_{L}\left(d \mu^{*}\right)}^{2}$ from above.

$$
\|Q\|^{2}=\int|Q(\xi)|^{2} d \mu^{*}(\xi)=\left(\int_{\left|\xi-\xi_{0}\right|<\delta}+\int_{\left|\xi-\xi_{0}\right| \geq \delta}\right)|Q(\xi)|^{2} d \mu^{*}(\xi),
$$

Both $F$ and $P$ have a local maximum of 1 at $\xi_{0}$, so we see that

$$
\begin{aligned}
\int_{\left|\xi-\xi_{0}\right|<\delta}|Q(\xi)|^{2} d \mu^{*}(\xi) & \leq \sup _{\left|\xi_{0}-\xi\right|<\delta} \frac{w^{*}(\xi)}{w(\xi)} \int_{\left|\xi_{0}-\xi\right|<\delta}\left|Q_{M}(\xi)\right|^{2} d \mu(\xi) \\
& \leq \sup _{\left|\xi_{0}-\xi\right|<\delta} \frac{w^{*}(\xi)}{w(\xi)} \lambda_{M}\left(\xi_{0}, \mu\right) .
\end{aligned}
$$

The measure $\mu^{*}$ is pure point on $\mathbb{R} \backslash \mathfrak{e}_{\delta_{1}}$ and the zeros of $P$ coincide with the mass points of $\mu^{*}$, so integrating $\left|F^{N} P\right|^{2}$ over the set $\mathfrak{e}_{\delta_{1}}$ is the same as the integrating over $\mathbb{R}$. We use (1.4.1) to show that the integral of $\left|Q^{2}\right|$ over $\left|\xi-\xi_{0}\right| \geq \delta$ is small for large $N$ :

$$
\begin{gathered}
\int_{\left|\xi-\xi_{0}\right| \geq \delta}|Q(\xi)|^{2} d \mu^{*}(\xi) \leq \frac{C \lambda_{M}\left(\xi_{0}\right) e^{4 \epsilon M}}{T} \int_{\left|\xi-\xi_{0}\right| \geq \delta, \xi \in \mathfrak{e}_{\delta_{1}}}\left|F(\xi)^{N} P(\xi)\right|^{2} d \mu^{*}(\xi) \\
\leq \frac{C \lambda_{M}\left(\xi_{0}\right) e^{4 \epsilon M}}{T}\left(\int_{\delta \leq\left|\xi-\xi_{0}\right| \leq 2}+\int_{\left|\xi-\xi_{0}\right|>2}\right)|F(\xi)|^{2 N} P^{2}(\xi) d \mu^{*}(\xi) .
\end{gathered}
$$

We have split the integral into two pieces: one that is close to $\xi_{0}$ and one that is far. For the close piece, since 1 is a maximum of $F$ on $\left[\xi_{0}-2, \xi_{0}+2\right]$ there exists $\gamma<1$ such that $F(\xi)<\gamma$ on $\left\{\xi: \delta<\left|\xi-\xi_{0}\right| \leq 2\right\}$. Therefore,

$$
\int_{\left\{\xi:\left|\xi-\xi_{0}\right| \leq 2\right\} \backslash\left[\xi_{0}-\delta, \xi_{0}+\delta\right]}|F(\xi)|^{2 N} P^{2}(\xi) d \mu^{*}(\xi) \leq C \gamma^{2 N}
$$

For the second piece,

$$
\int_{\left|\xi-\xi_{0}\right|>2}|F(\xi)|^{2 N} P^{2}(\xi) d \mu^{*}(\xi) \leq \int_{\left|\xi-\xi_{0}\right|>2} \frac{C \xi^{2 n+2} \xi_{0}}{\left(\xi-\xi_{0}\right)^{2 N}} d \mu^{*}(\xi) \leq C \xi_{0} 2^{-2 N}
$$

for $N>n+1$. The last bound follows from Lemma 3.3.2.

Since $\xi_{0} \in I \subset \mathfrak{e}^{i n t}$ for a compact interval $I$ and $\lambda_{M}\left(\xi_{0}\right)$ is continuous on $I$, we can 
choose $C$ that is uniform in $\xi_{0}$ on $I$ in Lemma 3.3.3.

We now prove Theorem 1.4.2

Suppose $d \mu^{*}, d \mu, I$ as in theorem and let $\xi(L) \rightarrow \xi_{0} \in I$.

Fix $\delta, \epsilon$. Let $\delta_{1}$ be small enough so that regularity bounds (1.4.1) hold for both $\mu, \mu^{*}$ on $E_{\delta_{1}}$ and let $n$ be the number of mass points of $\mu^{*}$ outside of $E_{\delta_{1}}$. Pick $N_{1}, N_{2}>(n+1) / \epsilon$ so that $(1 / 2)^{N_{1}}<e^{-4}$ and $\gamma^{N_{2}}<e^{-4}$. Let $N_{3}=\max \left\{N_{1}, N_{2}\right\}$ and $N=2 N_{3} M \epsilon$, so that Lemma 3.3.3 is applicable, and the sum of the second and third terms in (3.3.3) is $O\left(e^{-\epsilon M}\right)$. Divide by $\lambda_{L}\left(\xi_{0}, \mu\right)$ to get

$$
\frac{\lambda_{L}\left(\xi_{0}, \mu^{*}\right)}{\lambda_{L}\left(\xi_{0}, \mu\right)} \leq \sup _{\left|\xi-\xi_{0}\right|<\delta}\left(\frac{w^{*}(\xi)}{w(\xi)}\right) \frac{\lambda_{M}\left(\xi_{0}, \mu\right)}{\lambda_{L}\left(\xi_{0}, \mu\right)}+O\left(e^{-2 \epsilon M}\right) S_{L}\left(\xi_{0}, \xi_{0}, \mu\right)
$$

From regularity bounds (1.4.1) on $\mu$ and for fixed $N$, the second term on the right hand side tends to 0 as $M \rightarrow \infty$ :

$$
O\left(e^{-2 \epsilon M}\right) S_{L}\left(\xi_{0}, \xi_{0}, \mu\right) \leq O\left(e^{-2 \epsilon M}\right) C e^{\epsilon\left(M+\frac{\pi}{4 \xi_{0}} N\right)}=O\left(e^{-\epsilon M}\right)
$$

Then we take $\inf _{\left|\xi-\xi_{0}\right|<\delta}$ on both sides of (3.3.9) and we adjust the sup accordingly to get

$$
\inf _{\left|\xi-\xi_{0}\right|<\delta} \frac{\lambda_{L}\left(\xi, \mu^{*}\right)}{\lambda_{L}(\xi, \mu)} \leq \sup _{\left|\xi-\xi_{0}\right|<2 \delta}\left(\frac{w^{*}(\xi)}{w(\xi)}\right) \inf _{\left|\xi-\xi_{0}\right|<\delta} \frac{\lambda_{M}(\xi, \mu)}{\lambda_{L}(\xi, \mu)} .
$$

We then let $\delta \rightarrow 0$, then $M \rightarrow \infty$, and then $\epsilon \rightarrow 0$. We get by continuity and positivity of $w$ that

$$
\liminf _{L \rightarrow \infty} \frac{\lambda_{L}\left(\xi(L), \mu^{*}\right)}{\lambda_{L}(\xi(L), \mu)} \leq \frac{w^{*}\left(\xi_{0}\right)}{w\left(\xi_{0}\right)}
$$

To get the opposite inequality, we can interchange $\mu$ and $\mu^{*}$ in (3.3.3), use the corresponding $N$ given by the same formula, and divide by $\lambda_{L}\left(\xi_{0}, \mu^{*}\right)$.

All arguments given are uniform in $\xi_{0} \in I$. 


\subsection{Calculation of the reproducing kernel in the case of a periodic potential}

As in Gesztesy-Zinchenko $((2.8)$ of $[\mathrm{GZ06}])$, for $z \in \mathbb{C} \backslash \mathbb{R}$ let $\psi(z,-) \in L^{2}$, with $\psi(z, 0)=1$. Then the $m$-function is given by

$$
\psi(z, x)=y(z, x)-m(z) u(z, x)
$$

Similarly let $\tilde{\psi}$ be the $L^{2}$ solution with $\tilde{\psi}^{\prime}(z, 0)=1$. Then the corresponding $m$ function is given by

$$
\tilde{\psi}(z, x)=u(z, x)+\tilde{m} y(z, x) .
$$

Theorem 3.4.1. Let $A^{\#}=-\frac{d^{2}}{d x^{2}}+p$ be a Schrödinger operator with continuous periodic potential $p$ and either the Neumann or the Dirichlet boundary condition, and let $\rho(\xi) d \xi$ be its density of states. Let $\xi_{0} \in I \subset \sigma_{\mathrm{ess}}\left(A^{\#}\right)^{\text {int }}$, where $I$ is a closed and bounded interval. Then for $a, b \in \mathbb{R}$ uniformly in $I$

(1)

$$
\lim _{L \rightarrow \infty} \frac{S_{L}\left(\xi_{0}, \xi_{0}\right)}{\pi L}=\frac{\rho\left(\xi_{0}\right)}{w\left(\xi_{0}\right)}
$$

and

(2)

$$
\frac{S_{L}\left(\xi_{0}+\frac{a}{L}, \xi_{0}+\frac{b}{L}\right)}{S_{L}\left(\xi_{0}, \xi_{0}\right)}=\frac{\sin \left(\pi \rho\left(\xi_{0}\right)(b-a)\right)}{\pi \rho\left(\xi_{0}\right)(b-a)} .
$$

(3) Furthermore, (1.4.4) is satisfied.

Proof. The methods used here are similar to [Sim08b].

(1) We first show convergence then uniformity. We use the well known formula relating the $\rho(\xi)$ and $\Im G$, where $G$ is the Green's function. Gesztesy-Zinchenko 
((2.18) of [GZ06]) gives the Green's function explicitly, so we compute:

$$
\begin{aligned}
\rho(\xi) & =\lim _{L \rightarrow \infty} \frac{1}{L} \lim _{\epsilon \downarrow 0} \int_{0}^{L} \Im(G(x, x, \xi+i \epsilon)) d x \\
& =\lim _{L \rightarrow \infty} \frac{1}{L} \lim _{\epsilon \downarrow 0} \int_{0}^{L} \Im(u(\xi+i \epsilon, x) \psi(\xi+i \epsilon, x)) d x \\
& =\lim _{L \rightarrow \infty} \frac{1}{L} \lim _{\epsilon \downarrow 0} \Im m(\xi+i \epsilon) \int_{0}^{L} u(\xi, x)^{2} d x \\
& =\lim _{L \rightarrow \infty} \frac{w(\xi)}{\pi L} \int_{0}^{L} u(\xi, x)^{2} d x .
\end{aligned}
$$

Now, $\lim _{\epsilon \downarrow 0} \Im m(\xi+i \epsilon)=w(\xi)$ a.e., so the equality holds a.e..

We use continuity to show equality everywhere and uniformity of convergence. We let $\xi \in I \subset \mathfrak{e}^{i n t}$ and $f(\xi, x)=e^{i \theta(\xi) x} \phi(\xi, x)$ be the Floquet solution normalized so that $f^{\prime}(\xi, 0)=1$. Here $\phi$ is periodic in $x$ as in [MW66]. Then $f(\xi, 0) \notin \mathbb{R}$, and we claim that

$$
u(\xi, x)=\frac{f(\xi, x)-\overline{f(\xi, x)}}{f(\xi, 0)-\overline{f(\xi, 0)}} .
$$

Since $f, \bar{f}$ are solutions of the eigenvalue equation, so is the right hand side of (3.4.5). Therefore it suffices to check that the right hand side satisfies the Neumann boundary conditions, and it does.

Let

$$
g(\xi, x)=\frac{\phi(\xi, x)}{f(\xi, 0)-\overline{f(\xi, 0)}} .
$$

Then

$$
u(\xi, x)=e^{i \theta(\xi) x} g(\xi, x)+e^{-i \theta(\xi) x} \overline{g(\xi, x)} .
$$

The Wronskian of $e^{i \theta(\xi) x} g(\xi, x)$ and $e^{-i \theta(\xi) x} \overline{g(\xi, x)}$ is

$$
W(\xi)=-2 i g(\xi, x) \overline{g(\xi, x)} \theta(\xi)-g(\xi, x) \overline{g^{\prime}(\xi, x)}+\overline{g(\xi, x)} g^{\prime}(\xi, x)
$$

Substituting (3.4.7) for $u$ in the continuous analogue of the Christoffel-Darboux 
formula (3.2.6), we get that

$$
S_{L}(\xi, \xi)=2 \theta^{\prime}(\xi) i L W(\xi)+O(1)
$$

where $O(1)$ is bounded uniformly in $\xi \in I$ and $L$. Both $2 \theta^{\prime}(\xi) i W(\xi)$ and $\frac{\pi \rho(\xi)}{w(\xi)}$ are continuous in $\xi$ and equal a.e., meaning that

$$
\lim _{L \rightarrow \infty} \frac{S_{L}(\xi, \xi)}{L}=2 \theta^{\prime}(\xi) i W(\xi)=\frac{\pi \rho(\xi)}{w(\xi)}
$$

for all $\xi \in I$. The convergence in (3.4.3) is uniform.

A similar argument yields the result for $S_{L}$ corresponding to the Dirichlet boundary condition.

(2) For the Floquet solution $f$ normalized so that $f^{\prime}(\xi, 0)=1$ we have

$$
f(\xi, P k+s)=f(\xi, s) e^{i k \theta(\xi)} .
$$

By analytic perturbation theory (e. g. Theorems XII.13 and XII.3 of [RS78]), $f$ is real analytic in $\theta$ for $\theta \in(0, \pi) \cup(\pi, 2 \pi)$ and at closed gaps i.e. $\theta=\pi$ and $\Delta^{\prime}(\theta)=0$. By Theorem XIII.89 of [RS78], $\xi(\theta)$ is analytic and $\xi^{\prime}(\theta) \neq 0$, which implies that $\theta(\xi)$ is analytic on the interiors of the bands. The function $\theta(\xi)$ is also analytic at $\xi_{0}$ if $\xi_{0}$ is a closed gap. To see this we take the derivative of the discriminant equation $\Delta(\xi)=2 \cos (\theta)$ :

$$
\left.\frac{d}{d \xi}(\Delta(\xi))\right)=\frac{d}{d \xi} D(\xi) \frac{d}{d \theta} \xi(\theta)=-2 \sin (\theta)
$$

At a closed gap $\xi_{0}$, the right hand side has a single zero and $\frac{d}{d \xi} D(\xi)$ also has a single zero. This implies that $\frac{d}{d \theta} \xi(\theta) \neq 0$ at a closed gap so that $\theta(\xi)$ is analytic at $\xi_{0}$.

We can therefore take the Taylor series of $\theta(\xi), f(\xi, s)$, and $f^{\prime}(\xi, s)$ to get

$$
\begin{gathered}
f\left(\xi_{0}+\frac{a}{L}, x\right)=\left(f\left(\xi_{0}, s\right)+O\left(\frac{1}{L}\right)\right) e^{i k\left(\theta\left(\xi_{0}\right)+\frac{a \theta^{\prime}\left(\xi_{0}\right)}{L}+O\left(\frac{1}{L^{2}}\right)\right)}, \\
\frac{d}{d x} f\left(\xi_{0}+\frac{a}{L}, x\right)=\left(\frac{d}{d s} f\left(\xi_{0}, s\right)+O\left(\frac{1}{L}\right)\right) e^{i k\left(\theta\left(\xi_{0}\right)+\frac{a \theta^{\prime}\left(\xi_{0}\right)}{L}+O\left(\frac{1}{L^{2}}\right)\right)} .
\end{gathered}
$$


Letting $L=P k+s$, we substitute this into (3.4.5) to get

$$
\begin{aligned}
& 2 u\left(\xi_{0}+\frac{a}{L}, L\right) \Im f\left(\xi_{0}+\frac{a}{L}, 0\right)= \\
& \left(f\left(\xi_{0}, s\right)+O\left(\frac{1}{L}\right)\right) e^{i k\left(\theta\left(\xi_{0}\right)+\frac{a \theta^{\prime}\left(\xi_{0}\right)}{L}+O\left(\frac{1}{L^{2}}\right)\right)}-\overline{\left(f\left(\xi_{0}, s\right)+O\left(\frac{1}{L}\right)\right)} e^{-i k\left(\theta\left(\xi_{0}\right)+\frac{a \theta^{\prime}\left(\xi_{0}\right)}{L}+O\left(\frac{1}{L^{2}}\right)\right)} .
\end{aligned}
$$

We then compute:

$$
\begin{aligned}
& 4 \Im\left(f\left(\xi_{0}+\frac{a}{L}, 0\right) \Im\left(f\left(\xi_{0}+\frac{b}{L}, 0\right)\right) u\left(\xi_{0}+\frac{a}{L}, L\right) u^{\prime}\left(\xi_{0}+b / L, L\right)-u\left(\xi_{0}+b / L, L\right) u^{\prime}(\xi+a / L, L)\right. \\
& =\left(\left(f\left(\xi_{0}, s\right)+O\left(\frac{1}{L}\right)\right) e^{i n\left(\theta\left(\xi_{0}\right)+\frac{a \theta^{\prime}\left(\xi_{0}\right)}{L}+O\left(L^{-2}\right)\right)}-\overline{\left(f\left(\xi_{0}, s\right)+O\left(\frac{1}{L}\right)\right)} e^{-i n\left(\theta\left(\xi_{0}\right)+\frac{a \theta^{\prime}\left(\xi_{0}\right)}{L}+O\left(L^{-2}\right)\right)}\right) \\
& \times\left(\left(f^{\prime}\left(\xi_{0}, s\right)+O\left(\frac{1}{L}\right)\right) e^{i n\left(\theta\left(\xi_{0}\right)+\frac{b \theta^{\prime}\left(\xi_{0}\right)}{L}+O\left(L^{-2}\right)\right)}-\overline{\left(f^{\prime}\left(\xi_{0}, s\right)+O\left(\frac{1}{L}\right)\right)} e^{-i n\left(\theta\left(\xi_{0}\right)+\frac{b \theta^{\prime}\left(\xi_{0}\right)}{L}+O\left(L^{-2}\right)\right)}\right) \\
& -\left(\left(f\left(\xi_{0}, s\right)+O\left(\frac{1}{L}\right)\right) e^{i n\left(\theta\left(\xi_{0}\right)+\frac{b \theta^{\prime}\left(\xi_{0}\right)}{L}+O\left(L^{-2}\right)\right)}-\overline{\left(f\left(\xi_{0}, s\right)+O\left(\frac{1}{L}\right)\right)} e^{-i n\left(\theta\left(\xi_{0}\right)+\frac{b \theta^{\prime}\left(\xi_{0}\right)}{L}+O\left(L^{-2}\right)\right)}\right) \\
& \times\left(\left(f^{\prime}\left(\xi_{0}, s\right)+O\left(\frac{1}{L}\right)\right) e^{i n\left(\theta\left(\xi_{0}\right)+\frac{a \theta^{\prime}\left(\xi_{0}\right)}{L}+O\left(L^{-2}\right)\right)}-\overline{\left(f^{\prime}\left(\xi_{0}, s\right)+O\left(\frac{1}{L}\right)\right)} e^{-i n\left(\theta\left(\xi_{0}\right)+\frac{a \theta^{\prime}\left(\xi_{0}\right)}{L}+O\left(L^{-2}\right)\right)}\right) \\
& =-e^{i k\left(\frac{b-a}{L} \theta^{\prime}\left(\xi_{0}\right)+O\left(L^{-2}\right)\right)}\left(f^{\prime}\left(\xi_{0}, s\right) \overline{f\left(\xi_{0}, s\right)}+O\left(\frac{1}{L}\right)\right) O\left(\frac{1}{L}\right)-e^{i k\left(\frac{a-b}{L} \theta^{\prime}\left(\xi_{0}\right)+O\left(L^{-2}\right)\right)}\left(f\left(\xi_{0}\right) \overline{f^{\prime}\left(\xi_{0}\right)}+O\left(\frac{1}{L}\right)\right) \\
& +e^{i k\left(\frac{b-a}{L} \theta^{\prime}\left(\xi_{0}\right)+O\left(L^{-2}\right)\right)}\left(f\left(\xi_{0}, s\right) \overline{f^{\prime}\left(\xi_{0}\right)}+O\left(\frac{1}{L}\right)\right)+e^{i k\left(\frac{a-b}{L} \theta^{\prime}\left(\xi_{0}\right)+O\left(L^{-2}\right)\right)}\left(f^{\prime}\left(\xi_{0}\right) \overline{f\left(\xi_{0}\right)}+O\left(\frac{1}{L}\right)\right) \\
& =\left(f^{\prime}\left(\xi_{0}, s\right) \overline{f\left(\xi_{0}, s\right)}-\overline{f^{\prime}\left(\xi_{0}, s\right)} f\left(\xi_{0}, s\right)\right)\left(e^{i k\left(\frac{a-b}{L} \theta^{\prime}\left(\xi_{0}\right)+O\left(L^{-2}\right)\right)}-e^{i k\left(\frac{b-a}{L} \theta^{\prime}\left(\xi_{0}\right)+O\left(L^{-2}\right)\right)}\right) \\
& =W(f, \bar{f}) 2 i \sin \left(i\left(\frac{a-b}{P} \theta^{\prime}\left(\xi_{0}\right)+O\left(L^{-1}\right)\right)\right)
\end{aligned}
$$

where $W$ is the Wronskian of $f, \bar{f}$. The Wronskian is constant in $x$, so it suffice to compute it at 0 :

$$
W(f, \bar{f})=f^{\prime}\left(\xi_{0}, 0\right) \overline{f\left(\xi_{0}, 0\right)}-\overline{f^{\prime}\left(\xi_{0}, 0\right)} f\left(\xi_{0}, 0\right)=2 \Im f\left(\xi_{0}, 0\right)
$$

Thus we have obtained by direct calculation that

$$
\begin{aligned}
& 2 \Im f\left(\xi_{0}+\frac{a}{L}, 0\right) \Im f\left(\xi_{0}+\frac{b}{L}, 0\right)\left(u\left(\xi_{0}+\frac{a}{L}, L\right) u^{\prime}\left(\xi_{0}+\frac{b}{L}, L\right)-u\left(\xi_{0}+\frac{b}{L}, L\right) u^{\prime}\left(\xi+\frac{a}{L}, L\right)\right) \\
& =W(f, \bar{f}) i \sin \left(\frac{a-b}{P} \theta^{\prime}\left(\xi_{0}\right)+O\left(L^{-1}\right)\right) .
\end{aligned}
$$


Then substituting into the left hand side of (3.4.4), we get

$$
\begin{aligned}
& \frac{S_{L}\left(\xi_{0}+\frac{a}{L}, \xi_{0}+\frac{b}{L}\right)}{S_{L}\left(\xi_{0}, \xi_{0}\right)}= \\
& =\lim _{L \rightarrow \infty} \frac{w\left(\xi_{0}\right) \Im\left(f\left(\xi_{0}, 0\right)\right)\left(u\left(L, \xi_{0}+\frac{a}{L}\right) u^{\prime}\left(L, \xi_{0}+\frac{b}{L}\right)-u\left(L, \xi_{0}+\frac{b}{L}\right) u^{\prime}\left(L, \xi+\frac{a}{L}\right)\right)}{\Im\left(f\left(\xi_{0}+\frac{a}{L}, 0\right)\right) \Im\left(f\left(\xi_{0}+\frac{b}{L}, 0\right)\right) \rho\left(\xi_{0}\right)(b-a)} \\
& =\frac{\sin \left(\pi \rho\left(\xi_{0}\right)(b-a)\right)}{\pi \rho\left(\xi_{0}\right)(b-a)} .
\end{aligned}
$$

Here we have used that

$$
w(\xi)=\Im f(\xi, 0)
$$

which we get by substituting

$$
W(\xi)=\frac{f(0) \overline{f^{\prime}(0)}-f^{\prime}(0) \overline{f(0)}}{(2 i \Im f(\xi, 0))^{2}}=(2 i \Im f(\xi, 0))^{-1}
$$

in (3.4.9).

An identical calculation yields the result for the Dirichlet boundary condition.

To show (1.4.4), let $\epsilon(L) \rightarrow 0$ as $L \rightarrow \infty$. Since $u$ is real analytic in $\xi$,

$$
u^{2}(\xi+\epsilon(L), x)=u^{2}(\xi, x)+\frac{d}{d \xi}\left(u^{2}(\xi, x)\right) \epsilon(L)+o(\epsilon(L)),
$$

and since $I$ is compact, $\frac{d}{d \xi}\left(u^{2}(\xi, x)\right)$ achieves a maximum, so that $u^{2}(\xi+\epsilon(L), x)=$ $u^{2}(\xi, x)+O(\epsilon(L))$ uniformly on $I$. Thus,

$$
\begin{aligned}
\lim _{L \rightarrow \infty} \frac{w(\xi)}{\pi L} & \int_{0}^{L} u(\xi+\epsilon(L), x)^{2} d x= \\
& =\lim _{L \rightarrow \infty} \frac{w(\xi)}{\pi L} \int_{0}^{L} u(\xi, x)^{2} d x+O(\epsilon(L)) .
\end{aligned}
$$




\subsection{Off-Diagonal Kernel and Clock Behavior}

The main goal of this section is to prove our main result Theorem 1.3.1. We start by proving Lubinsky's inequality, which is similar to the discrete case:

Lemma 3.5.1. Let two measures $d \mu(\xi), d \mu^{*}(\xi)$ with $d \mu(\xi) \leq d \mu^{*}(\xi)$ be unnormalized spectral measures of Schrödinger operators. Then for any $\xi, \beta \in \mathbb{R}$,

$$
\frac{\left|S_{L}(\xi, \beta, \mu)-S_{L}\left(\xi, \beta, \mu^{*}\right)\right|}{S_{L}(\xi, \xi, \mu)} \leq\left(\frac{S_{L}(\beta, \beta, \mu)}{S_{L}(\xi, \xi, \mu)}\right)^{1 / 2}\left(1-\frac{S_{L}\left(\xi, \xi, \mu^{*}\right)}{S_{L}(\xi, \xi, \mu)}\right)^{1 / 2}
$$

Proof. The proof carries over from [Lub09]. Expanding,

$$
\begin{aligned}
& \int\left(S_{L}(\xi, \zeta, \mu)-S_{L}\left(\xi, \zeta, \mu^{*}\right)\right)^{2} d \mu(\zeta)= \\
& =\int S_{L}(\xi, \zeta, \mu)^{2} d \mu(\zeta)-2 \int S_{L}(\xi, \zeta, \mu) S_{L}\left(\xi, \zeta, \mu^{*}\right) d \mu(\zeta)+\int S_{L}^{2}\left(\xi, \zeta, \mu^{*}\right) d \mu(\zeta) \\
& =S_{L}(\xi, \xi, \mu)-2 S_{L}\left(\xi, \xi, \mu^{*}\right)+\int S_{L}\left(\xi, \zeta, \mu^{*}\right) d \mu(\zeta) .
\end{aligned}
$$

Since $d \mu \leq d \mu^{*}$

$$
\int S_{L}\left(\xi, \zeta, \mu^{*}\right) d \mu(\zeta) \leq \int S^{2}\left(\xi, \zeta, \mu^{*}\right) d \mu^{*}(\zeta)=S_{L}^{*}(\xi, \xi)
$$

Therefore,

$$
\int\left(S_{L}(\xi, \zeta, \mu)-S_{L}\left(\xi, \zeta, \mu^{*}\right)\right)^{2} d \mu(\zeta) \leq S_{L}(\xi, \xi, \mu)-S_{L}\left(\xi, \xi, \mu^{*}\right)
$$

Using the variational principle for the Christoffel-Darboux symbol e.g. the minimizing property, for any $\pi(\zeta) \in H_{L}$ and any $\beta \in \mathbb{R}$

$$
S_{L}(\beta, \beta, \mu)^{-1} \leq \int \frac{\pi(\zeta)^{2}}{\pi(\beta)^{2}} d \mu(\zeta)
$$

Using $\pi(\zeta)=S_{L}(\xi, \zeta, \mu)-S_{L}\left(\xi, \zeta, \mu^{*}\right)$ we get that

$$
\left|S_{L}(\xi, \beta, \mu)-S_{L}\left(\xi, \beta, \mu^{*}\right)\right| \leq S_{L}(\beta, \beta, \mu)^{1 / 2}\left(S_{L}\left(\xi, \xi, \mu^{*}\right)-S_{L}\left(\xi, \xi, \mu^{*}\right)\right) .
$$


We then show

Lemma 3.5.2. Let $d \mu, d \mu^{*}$ be unnormalized spectral measures with $\sigma_{\mathrm{ess}}(d \mu)=\sigma_{\mathrm{ess}}\left(d \mu^{*}\right)$. If $d \mu(\xi)$ obeys regularity bounds and $d \mu(\xi) \leq d \mu^{*}(\xi)$ then $d \mu^{*}(\xi)$ also obeys regularity bounds.

Proof. Since $d \mu \leq d \mu^{*},\|Q\|_{d \mu} \leq\|Q\|_{d \mu^{*}}$ for all $Q \in L^{2}(d \mu) \cap L^{2}\left(d \mu^{*}\right)$, so

$$
\begin{aligned}
& \inf \left\{\|Q\|_{d \mu}: Q\left(\xi_{0}\right)=1, Q(\xi)=\int_{0}^{L} f(x) \cos (\sqrt{\xi} x) d x\right\} \\
\leq & \inf \left\{\|Q\|_{d \mu^{*}}: Q\left(\xi_{0}\right)=1, Q(\xi)=\int_{0}^{L} f(x) \cos (\sqrt{\xi} x) d x\right\} .
\end{aligned}
$$

By the variational principle, this implies that $\lambda_{L}(\xi, \mu) \leq \lambda_{L}\left(\xi, \mu^{*}\right)$. If $u, u^{*}$ are the solutions of the eigenvalue equations corresponding to $d \mu, d \mu^{*}$ respectively, then

$$
C e^{\epsilon L} \geq \int_{0}^{L} u(\xi, x)^{2} d x \geq \int_{0}^{L} u^{*}(\xi, x)^{2} d x
$$

We now prove Theorem 1.3.1.

Proof. Let $A=-\frac{d^{2}}{d x^{2}}+p(x)+q(x)$ and $A^{\#}=-\frac{d^{2}}{d x^{2}}+p(x)$ be Schrödinger operators with periodic continuous $p$ and non-destructive zero-average $q$ (Definition 1.2.2). Suppose the corresponding spectral measures $d \mu, d \mu^{\#}$ satisfy regularity bounds. Suppose there exists a closed and bounded interval $I \subset \sigma_{\text {ess }}(A)^{\text {int }}$ such that $\xi_{0} \in I, w$ is absolutely continuous and positive on $I$, and $\left(\sigma_{\text {ess }}\left(d \mu_{s}\right) \cup \sigma_{\text {ess }}\left(d \mu_{s}^{\#}\right)\right) \cap I=\emptyset$.

Let $s>0$ such that $s w^{\#}\left(\xi_{0}\right)=w\left(\xi_{0}\right)$. From $\mu$, $\mu^{\#}$ we construct a new unnormalized spectral measure $\mu^{*}$ which dominates $\mu, s \mu^{\#}$ and is absolutely continuous on $I$ with $w^{*}\left(\xi_{0}\right)=w\left(\xi_{0}\right)$. Let $d \mu^{*}(\xi)=\sup \left\{s d \mu^{\#}(\xi), d \mu(\xi)\right\}$, for $\xi<R$, and $d \mu^{*}(\xi)=s d \mu^{\#}(\xi)+d \mu(\xi)$, for $\xi \geq R$, where $R \in \mathbb{R}$ with $I \subset(-\infty, R)$. We claim that $\mu^{*}$ is an unnormalized spectral measure. 
A measure $d \nu$ is a spectral measure for a boundary value problem (Theorem 2.3.1 of [Mar86]) if and only if

(1) The functional on $H_{L}$ given by the inner product $\langle-, \pi(\xi)\rangle_{d \nu}$ is non-trivial for all non-trivial $\pi$.

(2) The function

$$
\Phi(x, \nu)=\int \frac{1-\cos (\sqrt{\xi} x)}{\xi} d \nu(\xi)
$$

is thrice continuously differentiable in $x$ and $\Phi^{\prime}(0+, \nu)=1$.

Condition (1) is true for $d \mu^{*}$, since it is true for both $\mu$ and $\mu^{\#}$. To show condition $(2)$, let $\Phi_{R}(x, \nu)=\int_{-\infty}^{R} \frac{1-\cos (\sqrt{\xi} x)}{\xi} d \nu$, for any locally finite measure $d \nu$. Then $\Phi_{R}(x, \mu)$, $\Phi_{R}\left(x, \mu^{\#}\right), \Phi_{R}\left(x, \mu^{*}\right)$ are in $C^{\infty}$ by Dominated Convergence Theorem and

$$
\int_{R}^{\infty} \frac{1-\cos (\sqrt{\xi} x)}{\xi} d \mu^{*}=\Phi(x, \mu)-\Phi_{R}(x, \mu)+\Phi\left(x, \mu^{\#}\right)-\Phi_{R}\left(x, \mu^{\#}\right)
$$

is in $C^{3}$ as a sum of $C^{3}$ functions, making $\Phi\left(x, \mu^{*}\right) \in C^{3}$. By continuity of $\Phi_{R}^{\prime}(x)$ and the Dominated Convergence Theorem

$$
\Phi_{R}^{\prime}\left(0+, \mu^{*}\right)=\Phi_{R}^{\prime}\left(0, \mu^{*}\right)=\int_{0}^{R} \frac{\sin (0)}{\sqrt{\xi}} d \mu^{*}(\xi)=0
$$

SO

$$
\Phi^{\prime}\left(0+, \mu^{*}\right)=\Phi^{\prime}(0+, \mu)+\Phi^{\prime}\left(0+, \mu^{\#}\right)=1+s .
$$

Thus, dividing $d \mu^{*}$ by $1+s$ will yield a spectral measure. Additionally, the boundary condition of $d \mu^{*}$ is the same as that for $d \mu, d \mu^{\#}$ (Theorem 2.4.2 of Marchenko [Mar86]).

By Lemma 3.5.2 above, $\mu^{*}$ obeys the regularity bound. Thus, by (1.4.2)

$$
\frac{S_{L}\left(\xi_{0}+a / L, \xi_{0}+a / L, \mu\right)}{S_{L}\left(\xi_{0}+b / L, \xi_{0}+b / L, \mu^{*}\right)} \rightarrow 1
$$

and

$$
\frac{S_{L}\left(\xi_{0}+a / L, \xi_{0}+a / L, s \mu^{\#}\right)}{S_{L}\left(\xi_{0}+b / L, \xi_{0}+b / L, \mu^{*}\right)} \rightarrow 1
$$


Dividing by $S_{L}\left(\xi_{0}, \xi_{0}\right)$ and applying Lubinsky's inequality, we get that

$$
\begin{aligned}
& \frac{\left|S_{L}\left(\xi_{0}+\frac{a}{L}, \xi_{0}+\frac{b}{L}, \mu\right)-S_{L}\left(\xi_{0}+\frac{a}{L}, \xi_{0}+\frac{b}{L}, \mu^{*}\right)\right|^{2}}{S_{L}\left(\xi_{0}+\frac{b}{L}, \xi_{0}+\frac{b}{L}, \mu^{*}\right)} \\
& \leq S_{L}\left(\xi_{0}+\frac{a}{L}, \xi_{0}+\frac{a}{L}, \mu\right)-S_{L}\left(\xi_{0}+\frac{a}{L}, \xi_{0}+\frac{a}{L}, \mu^{*}\right),
\end{aligned}
$$

and

$$
\begin{aligned}
& \frac{\left|S_{L}\left(\xi_{0}+\frac{a}{L}, \xi_{0}+\frac{b}{L}, s \mu^{\#}\right)-S_{L}\left(\xi_{0}+\frac{a}{L}, \xi_{0}+\frac{b}{L}, \mu^{*}\right)\right|^{2}}{S_{L}\left(\xi_{0}+\frac{b}{L}, \xi_{0}+\frac{b}{L}, \mu^{*}\right)} \\
& \leq S_{L}\left(\xi_{0}+\frac{a}{L}, \xi_{0}+\frac{a}{L}, s \mu^{\#}\right)-S_{L}\left(\xi_{0}+\frac{a}{L}, \xi_{0}+\frac{a}{L}, \mu^{*}\right)
\end{aligned}
$$

which gives that

$$
\frac{S_{L}\left(\xi_{0}+\frac{a}{L}, \xi_{0}+\frac{b}{L}, \mu\right)}{S_{L}\left(\xi_{0}+\frac{a}{L}, \xi_{0}+\frac{b}{L}, s \mu^{\#}\right)} \rightarrow 1
$$

Since

$$
\frac{S_{L}\left(\xi_{0}, \xi_{0}, \mu\right)}{S_{L}\left(\xi_{0}, \xi_{0}, s \mu^{\#}\right)} \rightarrow 1
$$

we get that

$$
\lim _{L \rightarrow \infty} \frac{S_{L}\left(\xi_{0}+\frac{a}{L}, \xi_{0}+\frac{b}{L}, \mu\right)}{S_{L}\left(\xi_{0}, \xi_{0}, \mu\right)}=\lim _{L \rightarrow \infty} \frac{S_{L}\left(\xi_{0}+\frac{a}{L}, \xi_{0}+\frac{b}{L}, s \mu^{\#}\right)}{S_{L}\left(\xi_{0}, \xi_{0}, s \mu^{\#}\right)}
$$

The limit on the right is equal to (1.3.1) and all limits are uniform on $I$ and $|a|,|b|<B$.

Like [Sim08b], [LL08], we can now deduce clock spacing of the zeros for a perturbed periodic potential. Here we prove Corollary 1.3.3.

Proof. Fix an interval $I \subset \mathfrak{e}^{\text {int }}$ and $\xi^{*} \in I$. We want to show uniform clock behavior at $\xi^{*}$ of zeros of $u^{\prime}$ and $y$ in $\xi$ as $L$ gets large. More precisely, if $\xi_{n}$ is a successive numbering of zeros with $\ldots \xi_{-1}<\xi^{*} \leq \xi_{0}<\xi_{1}<\ldots$, then

$$
\lim _{L} L\left|\left(\xi_{n}-\xi_{n+1}\right)\right| \rho\left(\xi^{*}\right)=1
$$


By the Christoffel-Darboux formula (3.2.4),

$$
\frac{u\left(\xi^{*}, L\right)}{u^{\prime}\left(\xi^{*}, L\right)}=\frac{u\left(\xi^{*}+a / L, L\right)}{u^{\prime}\left(\xi^{*}+a / L, L\right)}
$$

for $a \neq 0$ if and only if $S_{L}\left(\xi^{*}, \xi^{*}+a / L\right)=0$. From (1.4.5) and (3.4.3) we see that $S_{L}\left(\xi^{*}, \xi^{*}\right)=O(L)$. Now, by (1.3.1) and since $S_{L}\left(\xi^{*}, \xi^{*}\right)=O(L), S_{L}\left(\xi^{*}, \xi^{*}+a / L\right)=$ $o(1 / L)$ if and only if $a=\frac{k}{\rho\left(\xi^{*}\right)}+o(1 / L)$. The convergence in $L$ is uniform on $I$, since (1.3.1) is uniform on $I$. The argument is the same for $y$.

\subsection{Example: the Free Schrödinger Operator}

The arguments in Section 3.1 apply also to non-destructive zero-average perturbations of the free Schrödinger operator, thus giving us the regularity bounds condition. We know the spectral measure for the free Schrödinger operator [Tes09], and it is indeed continuous and non-negative on $[0, \infty)$. The solution of the eigenvalue equation for the free Schrödinger operator

$$
-\frac{d^{2}}{d x^{2}} u(x, \xi)=\xi u(x, \xi)
$$

with the Neumann boundary condition is $\cos (\sqrt{\xi} x)<e^{\epsilon x}$ on $[0, \infty)$. We compute $S_{L}(\xi, \beta)$ and $S_{L}(\xi, \xi)$ directly:

$$
S_{L}(\xi, \beta)=\int_{0}^{L} \cos (\sqrt{\xi} x) \cos (\sqrt{\beta} x) d x=\frac{\sin ((\sqrt{\xi}-\sqrt{\beta}) L)}{2(\sqrt{\xi}-\sqrt{\beta})}+\frac{\sin ((\sqrt{\xi}+\sqrt{\beta}) L)}{2(\sqrt{\xi}+\sqrt{\beta})},
$$

and

$$
S_{L}(\xi, \xi)=\frac{L}{2}+\frac{\sin (2 \sqrt{\xi} L)}{4 \sqrt{\xi}} .
$$

Then model property (3) is clear and we check property (4):

$$
\limsup _{\epsilon \rightarrow 0} \limsup _{L \rightarrow \infty} \frac{\frac{L+\epsilon L}{2}+\frac{\sin (2 \sqrt{\xi}(L+\epsilon L)}{4 \sqrt{\xi}}}{\frac{L}{2}+\frac{\sin (2 \sqrt{\xi} L)}{4 \sqrt{\xi}}}=1
$$


Locally at $\xi_{0}$ we get

$$
\lim _{L \rightarrow \infty} \frac{S_{L}\left(\xi_{0}+a / L, \xi_{0}+b / L\right)}{S_{L}\left(\xi_{0}, \xi_{0}\right)}=\frac{2 \sqrt{\xi_{0}} \sin \left(\frac{a-b}{2 \sqrt{\xi_{0}}}\right)}{a-b}
$$

This coincides with (3.4.3), since the density of states for the free Schrödinger operator is

$$
\rho(\xi)=(2 \pi)^{-1} \xi^{-1 / 2}
$$

for $\xi \in[0, \infty)$ (Example 8.1 of [BS91]). 


\section{Bibliography}

[BS91] F. A. Berezin and M. A. Shubin. The Schrödinger equation, volume 66 of Mathematics and its Applications (Soviet Series). Kluwer Academic Publishers Group, Dordrecht, 1991. Translated from the 1983 Russian edition by Yu. Rajabov, D. A. Leĭtes and N. A. Sakharova and revised by Shubin, With contributions by G. L. Litvinov and Leŭtes.

[CL55] Earl A. Coddington and Norman Levinson. Theory of ordinary differential equations. McGraw-Hill Book Company, Inc., New York-Toronto-London, 1955 .

[CS77] K. Chadan and P. C. Sabatier. Inverse problems in quantum scattering theory. Springer-Verlag, New York, 1977. With a foreword by R. G. Newton, Texts and Monographs in Physics.

[ET55] P. Erdös and P. Turán. On the role of the Lebesgue functions in the theory of the Lagrange interpolation. Acta Math. Acad. Sci. Hungar., 6:47-66, 1955.

[Fre71] G. Freud. Orthogonal Polynomials. Pergamon Press, Oxford, New York, 1971.

[GL55] I. M. Gel'fand and B. M. Levitan. On the determination of a differential equation from its spectral function. Amer. Math. Soc. Transl. (2), 1:253304, 1955. 
[GS00] Fritz Gesztesy and Barry Simon. A new approach to inverse spectral theory. II. General real potentials and the connection to the spectral measure. Ann. of Math. (2), 152(2):593-643, 2000.

[GZ06] Fritz Gesztesy and Maxim Zinchenko. On spectral theory for Schrödinger operators with strongly singular potentials. Math. Nachr., 279(9-10):1041$1082,2006$.

[Jos47] Res Jost. Über die falschen Nullstellen der Eigenwerte der S-Matrix. Helvetica Phys. Acta, 20:256-266, 1947.

[KV02] A. B. J. Kuijlaars and M. Vanlessen. Universality for eigenvalue correlations from the modified Jacobi unitary ensemble. Int. Math. Res. Not., (30):15751600, 2002.

[LL08] Eli Levin and Doron S. Lubinsky. Applications of universality limits to zeros and reproducing kernels of orthogonal polynomials. J. Approx. Theory, 150(1):69-95, 2008.

[LS08] Yoram Last and Barry Simon. Fine structure of the zeros of orthogonal polynomials. IV. A priori bounds and clock behavior. Comm. Pure Appl. Math., 61(4):486-538, 2008.

[Lub09] D. S. Lubinsky. A new approach to universality limits involving orthogonal polynomials. Annals of Mathematics, 170:915-939, 2009.

[Mar86] Vladimir A. Marchenko. Sturm-Liouville operators and applications, volume 22 of Operator Theory: Advances and Applications. Birkhäuser Verlag, Basel, 1986. Translated from the Russian by A. Iacob.

[Min96] Nariyuki Minami. Local fluctuation of the spectrum of a multidimensional Anderson tight binding model. Comm. Math. Phys., 177(3):709-725, 1996.

[Mol81] S. A. Molčanov. The local structure of the spectrum of the one-dimensional Schrödinger operator. Comm. Math. Phys., 78(3):429-446, 1980/81. 
[MW66] Wilhelm Magnus and Stanley Winkler. Hill's equation. Interscience Tracts in Pure and Applied Mathematics, No. 20. Interscience Publishers John Wiley \& Sons New York-London-Sydney, 1966.

[Rei04] Linda E. Reichl. The transition to chaos. Institute for Nonlinear Science. Springer-Verlag, New York, second edition, 2004. Conservative classical systems and quantum manifestations.

[RS78] Michael Reed and Barry Simon. Methods of modern mathematical physics. IV. Analysis of operators. Academic Press [Harcourt Brace Jovanovich Publishers], New York, 1978.

[Sim82] Barry Simon. Schrödinger semigroups. Bull. Amer. Math. Soc. (N.S.), $7(3): 447-526,1982$.

[Sim05] Barry Simon. Orthogonal polynomials on the unit circle. Part 1, volume 54 of American Mathematical Society Colloquium Publications. American Mathematical Society, Providence, RI, 2005. Classical theory.

[Sim08a] Barry Simon. The Christoffel-Darboux kernel. 'Perspectives in PDE, Harmonic Analysis and Applications,' a volume in honor of V.G. Maz'ya's 70th birthday, Proceedings of Symposia in Pure Mathematics, 79:295-335, 2008.

[Sim08b] Barry Simon. Two extensions of Lubinsky's universality theorem. J. Anal. Math., 105:345-362, 2008.

[ST92] Herbert Stahl and Vilmos Totik. General orthogonal polynomials, volume 43 of Encyclopedia of Mathematics and its Applications. Cambridge University Press, Cambridge, 1992.

[Sze75] Gábor Szegö. Orthogonal polynomials. American Mathematical Society, Providence, R.I., fourth edition, 1975. American Mathematical Society, Colloquium Publications, Vol. XXIII. 
[Tes09] Gerald Teschl. Mathematical Methods in Quantum Mechanics, volume 150 of Graduate Studies in Mathematics. American Mathematical Society, 2009.

[Tot] V. Totik. Universality and fine zero spacing on general sets. in preparation.

[Ull72] J. L. Ullman. On the regular behaviour of orthogonal polynomials. Proc. London Math. Soc. (3), 24:119-148, 1972.

[Wei87] Joachim Weidmann. Spectral theory of ordinary differential operators, volume 1258 of Lecture Notes in Mathematics. Springer-Verlag, Berlin, 1987. 